\title{
Discovery of Highly Blueshifted Broad Balmer and Metastable Helium Absorption Lines in a Tidal Disruption Event
}

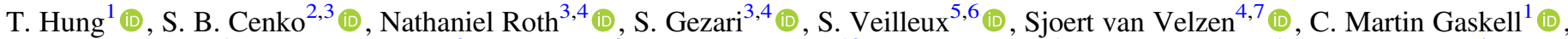 \\ Ryan J. Foley ${ }^{1}$, N. Blagorodnova ${ }^{8}$ (i), Lin Yan $^{9}$ (i), M. J. Graham ${ }^{10}{ }^{(10}$, J. S. Brown ${ }^{1}$ (i), M. R. Siebert ${ }^{1}$, Sara Frederick ${ }^{4}$ (1), \\ Charlotte Ward ${ }^{4}$, Pradip Gatkine ${ }^{4}$, Avishay Gal-Yam ${ }^{11}$ (i), Yi Yang ${ }^{11}$, S. Schulze ${ }^{11}$ (1), G. Dimitriadis ${ }^{1}$ (1), Thomas Kupfer ${ }^{12}$ (1)

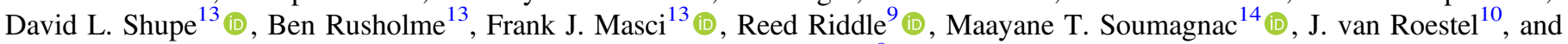 \\ Richard Dekany ${ }^{9}$ \\ ${ }^{1}$ Department of Astronomy and Astrophysics, University of California, Santa Cruz, CA 95064, USA \\ ${ }^{2}$ Astrophysics Science Division, NASA Goddard Space Flight Center, MC 661, Greenbelt, MD 20771, USA \\ ${ }^{3}$ Joint Space-Science Institute, University of Maryland, College Park, MD 20742, USA \\ ${ }^{4}$ Department of Astronomy, University of Maryland, College Park, MD 20742, USA \\ 5 Institute of Astronomy and Kavli Institute for Cosmology, Cambridge, UK \\ ${ }^{6}$ University of Cambridge, Cambridge CB3 OHA, UK \\ ${ }^{7}$ Center for Cosmology and Particle Physics, New York University, New York, NY 10003, USA \\ ${ }^{8}$ Department of Astrophysics/IMAPP, Radboud University, Nijmegen, The Netherlands \\ ${ }^{9}$ Caltech Optical Observatories, California Institute of Technology, Pasadena, CA 91125, USA
${ }^{10}$ Division of Physics, Mathematics, and Astronomy, California Institute of Technology, Pasadena, CA 91125, USA \\ ${ }^{11}$ Department of Particle Physics and Astrophysics, Weizmann Institute of Science, 234 Herzl St., Rehovot 76100, Israel \\ ${ }_{12}^{12}$ Kavli Institute for Theoretical Physics, University of California, Santa Barbara, CA 93106, USA \\ ${ }^{13}$ IPAC, California Institute of Technology, 1200 E. California Blvd., Pasadena, CA 91125, USA \\ ${ }^{14}$ Benoziyo Center for Astrophysics, Weizmann Institute of Science, Rehovot, Israel \\ Received 2019 March 11; revised 2019 May 16; accepted 2019 May 26; published 2019 July 15
}

\begin{abstract}
We report the discovery of nonstellar hydrogen Balmer and metastable helium absorption lines accompanying a transient, high-velocity $(0.05 c)$ broad absorption line (BAL) system in the optical spectra of the tidal disruption event (TDE) AT2018zr $(z=0.071)$. In the Hubble Space Telescope UV spectra, absorption of high- and lowionization lines is also present at this velocity, making AT2018zr resemble a low-ionization BALQSO. We conclude that these transient absorption features are more likely to arise in fast outflows produced by the TDE than absorbed by the unbound debris. In accordance with the outflow picture, we are able to reproduce the flat-topped $\mathrm{H} \alpha$ emission in a spherically expanding medium without invoking the typical prescription of an elliptical disk. We also report the appearance of narrow $\left(\sim 1000 \mathrm{kms}^{-1}\right) \mathrm{N}$ III $\lambda 4640$, He II $\lambda 4686, \mathrm{H} \alpha$, and $\mathrm{H} \beta$ emission in the latetime optical spectra of AT2018zr, which may be a result of UV continuum hardening at late times, as observed by Swift. Including AT2018zr, we find a high association rate (three out of four) of BALs in the UV spectra of TDEs. This suggests that outflows may be ubiquitous among TDEs and less sensitive to viewing angle effects compared to QSO outflows.
\end{abstract}

Key words: accretion, accretion disks - black hole physics - galaxies: nuclei - ultraviolet: general

\section{Introduction}

Occasionally, a star passing too close to a black hole may be disrupted by tidal stresses. This results in an observable transient flare of radiation powered by the accretion of about half of the stellar debris onto the black hole (Rees 1988; Phinney 1989). A significant amount of theoretical work has been devoted to predicting the rate at which the stellar debris falls back to the pericenter. For black holes with $M_{\mathrm{BH}} \lesssim$ $10^{7} M_{\odot}$, the fallback rate- and the accretion rate, if circularization is efficient-may exceed the Eddington rate at early times (Evans \& Kochanek 1989; Ulmer 1999; Strubbe \& Quataert 2009; Lodato \& Rossi 2011; Metzger \& Stone 2016; Wu et al. 2018), leading to the formation of radiation-powered winds or jets.

Observations across all wavelengths have revealed that outflows may be ubiquitous among tidal disruption events (TDEs). In the well-studied TDE ASASSN-14li, highly ionized outflows have been detected at both low (a few $\times 100 \mathrm{~km} \mathrm{~s}^{-1}$; Miller et al. 2015) and high ( $0.2 c$; Kara et al. 2018) velocities in the X-ray. Radio observations of ASASSN-14li also revealed the presence of a subrelativistic outflow (Alexander et al. 2016) or an off-axis relativistic jet (van Velzen et al. 2016; Pasham \& van Velzen 2018).

Spectroscopy is a powerful tool for probing the kinematics and physical conditions in TDEs. Especially in the far-UV (FUV), spectroscopy can shed light on the ionization structures owing to the wealth of atomic transitions encompassed in this wavelength range. For the three TDEs that were observed with the Space Telescope Imaging Spectrograph (STIS) on board the Hubble Space Telescope (HST), blueshifted absorption lines at FUV wavelengths that signify the presence of outflows were detected in all three sources, namely, ASASSN-14li, iPTF16fnl, and iPTF15af (Cenko et al. 2016; Blagorodnova et al. 2019; Brown et al. 2018). These absorption lines are thought to be "intrinsic," meaning that the absorbing gas is physically close to the TDE. In particular, the broad, saturated absorption troughs of high-ionization transitions in iPTF15af are reminiscent of those seen in broad absorption line (BAL) QSOs. Blagorodnova et al. (2019) concluded that these features could only form in absorbers with high column densities $N_{\mathrm{H}}>10^{23} \mathrm{~cm}^{-2}$. 
Although the current sample of UV spectroscopy of TDEs is small, a few spectroscopic distinctions between TDEs and QSOs have emerged in the past few years. For example, common quasar emission lines such as Mg II $\lambda \lambda 2796,2803$ and C III] $\lambda 1909$ are either weak or entirely missing in TDE spectra. The absence of Mg II $\lambda \lambda 2796,2803$ may be explained if these TDEs have a hotter continuum that has photoionized most $\mathrm{Mg}$ to higher ionization states (Cenko et al. 2016; Brown et al. 2018). On the other hand, the prominent N III] $\lambda 1750$ relative to C III] $\lambda 1909$ in TDEs may imply abundance anomalies due to the CNO cycle in the predisrupted star (Cenko et al. 2016; Kochanek 2016). Simulations have shown that the anomalous abundance features should be present after the time of peak fallback rate $\left(t_{\text {peak }}\right)$ and are more significant in higher-mass stars (Kochanek 2016; Yang et al. 2017; Gallegos-Garcia et al. 2018).

Observationally, TDEs have been discovered at a rate of one to two per year. We expect to see an order-of-magnitude increase in the TDE discovery rate from the combined yield of ground-based optical surveys since the beginning of 2018 (e.g., Hung et al. 2018). In light of this opportunity, we have started a monitoring campaign to obtain a series of UV spectra of newly discovered TDEs with HST. The first target of this campaign (Program ID 15331; PI: Cenko) is AT2018zr (aka PS18kh). In this paper, we present the analysis of the intensive spectroscopic observations of the TDE AT2018zr spanning across the UV and optical wavelengths in the first 3 months since discovery, plus two late-time ( $\Delta t=169$ and 248 days) optical spectra.

While inspecting the data, we discovered a high-velocity $(\sim 0.05 c)$ BAL system that is accompanied by nonstellar hydrogen Balmer and metastable helium absorption, the first time this has been observed in a TDE. The high signal-to-noise ratio $(\mathrm{S} / \mathrm{N})$ optical spectra enabled us to propose that a spherically expanding outflow is preferred over the elliptical disk model (Holoien et al. 2018) for generating the observed flat-topped $\mathrm{H} \alpha$ line.

This paper is structured as follows. We summarize the discovery and photometric observations of AT2018zr in Section 2, detail the observation configurations and data reduction for the UV and optical spectra in this work in Section 3, describe our analysis of the emission and absorption features identified in the data in Section 4, and compare these observations with other TDEs and discuss the results in Section 5. We summarize our findings in Section 6.

\section{Discovery of AT2018zr}

The TDE AT2018zr (aka PS18kh) was first discovered by Pan-STARRS1 on UT 2018 March 2 (Holoien et al. 2018; Tucker et al. 2018). The flare is coincident (offset $\lesssim 0$ "' 1 ) with the galaxy SDSS J075654.53+341543.6 at a redshift of $z=0.071$ (Section 3.2). Archival observations of this galaxy suggest that it is dominated by an old stellar population $\left(t_{\text {age }}=9.8 \mathrm{Gyr}\right)$ with a stellar mass of $5 \times 10^{9} M_{\odot}$ (van Velzen et al. 2019). The lack of X-ray emission prior to the TDE flare suggests little or no active galactic nucleus (AGN) activity (Holoien et al. 2018). A black hole mass of $\approx 10^{7} M_{\odot}$ is inferred from the host photometry (Holoien et al. 2018; van Velzen et al. 2019). While the Zwicky Transient Facility (ZTF; Bellm et al. 2019; Graham et al. 2019) was still in the commissioning phase, the survey serendipitously observed this object from 2018 February 7. A complete set of ZTF light curves of this source can be found in van Velzen et al. (2019).

Holoien et al. (2018) and van Velzen et al. (2019) analyzed the broadband multiwavelength (UV/optical/X-ray/radio) properties of AT2018zr. Before the object was Sun-constrained, they found that the UV and optical emission of this source corresponds to a constant blackbody temperature of $T \approx 1.4 \times 10^{4} \mathrm{~K}$ in the first 40 days, then increases to $T \approx 2.2 \times 10^{4} \mathrm{~K}$ (Holoien et al. 2018; van Velzen et al. 2019). A weak, thermal $(k T \sim 100 \mathrm{eV}) \mathrm{X}$-ray source 2 orders of magnitude less luminous than the UV was detected (van Velzen et al. 2019). The late-time ( $\Delta t \gtrsim 170$ days) UV/optical photometric observations show a significant increase in the blackbody temperature $\left(T \gtrsim 5 \times 10^{4} \mathrm{~K}\right)$, while the $\mathrm{X}$-ray flux remains almost the same (van Velzen et al. 2019).

While van Velzen et al. (2019) focused on the broadband optical-to-X-ray behavior of AT2018zr, Holoien et al. (2018) included analysis of the optical spectra of AT2018zr. Their observations were made in the first 3 months of discovery before the target became Sun-constrained. Their analysis used a combination of wind, elliptical disk, and spiral arm to fit the $\mathrm{H} \alpha$ line profile and inferred a large size for the accretion disk $\left(r_{\text {in }} \sim 500 r_{g}\right.$ and $\left.r_{\text {out }} \sim 15,000 r_{g}\right)$.

In this work, we present the first analysis of the multi-epoch HST UV spectra of AT2018zr in conjunction with optical spectroscopic observations obtained independently in the time frame of the observations in Holoien et al. (2018). Although our optical spectroscopic data set is similar to that in Holoien et al. (2018), we use a different model to interpret the emission line shape (Section 4.3.2) for the data obtained in the same time period. Furthermore, our optical spectroscopic observations extend to later epochs ( $\Delta t \gtrsim 170$ days) to study the late-time spectroscopic evolution of this TDE, which have not been reported before.

\section{Observations}

After it was confirmed that AT2018zr was bright in the UV from the Neil Gehrels Swift Observatory (Swift; Gehrels et al. 2004) observations, we triggered a series of spectroscopic follow-up observations with HST STIS, as well as other ground-based optical telescopes. No observations were made when the target went behind the Sun from 2018 June to August. We resumed following up this source when it became visible again in 2018 September. All the spectra presented in this paper have been corrected for galactic extinction using the Schlafly \& Finkbeiner (2011) dust map. Assuming a Cardelli et al. (1989) extinction curve, using $R_{\mathrm{C}}=3.1$ and $E(B-V)=$ $0.0404 \pm 0.0006$ at this position corresponds to a Galactic visual extinction of $A_{\mathrm{V}}=0.124 \mathrm{mag}$. No correction has been made for any internal extinction. Throughout this paper, we adopt a flat $\Lambda \mathrm{CDM}$ cosmology with $H_{0}=69.3 \mathrm{~km} \mathrm{~s}^{-1} \mathrm{Mpc}^{-1}$, $\Omega_{m}=0.29$, and $\Omega_{\Lambda}=0.71$ (Bennett et al. 2013). The time difference $(\Delta t)$ is expressed in rest-frame time with respect to the $r$-band maximum at MJD 58,194.49.

\subsection{HST STIS Spectra}

We obtained five epochs of UV spectra of AT2018zr with HST STIS (GO 15331; PI: Cenko) on 2018 April 11 ( $\Delta t=$ 23 days), 25 ( $\Delta t=36$ days), and $30(\Delta t=41$ days) and May $20(\Delta t=59$ days $)$ and 23 ( $\Delta t=62$ days). The spectra were obtained through a $52^{\prime \prime} \times 0$ "! 2 aperture. For the near-UV 
Table 1

Observing Details of the Optical Spectra of AT2018zr

\begin{tabular}{|c|c|c|c|c|c|}
\hline Obs. Date & Phase (days) & Telescope + Instrument & Slit Width & Grism/Grating & Exp. Time (s) \\
\hline 2018 Mar 7 & -10 & $\mathrm{P} 60+\mathrm{SEDm}$ & & $\mathrm{N} / \mathrm{A}$ & 1800 \\
\hline 2018 Mar 26 & 8 & $\mathrm{P} 60+\mathrm{SEDm}$ & & $\mathrm{N} / \mathrm{A}$ & 1800 \\
\hline 2018 Mar 27 & 9 & $\mathrm{P} 60+\mathrm{SEDm}$ & & $\mathrm{N} / \mathrm{A}$ & 2700 \\
\hline 2018 Mar 28 & 10 & WHT + ACAM & 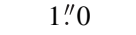 & V400 & 3200 \\
\hline 2018 Apr 4 & 16 & DCT + DeVeny & $1 . " 5$ & $300 \mathrm{~g} / \mathrm{mm}$ & 2400 \\
\hline 2018 Apr 11 & 23 & DCT + DeVeny & $1 " .5$ & $300 \mathrm{~g} / \mathrm{mm}$ & 2400 \\
\hline 2018 Apr 17 & 28 & Keck I + LRIS & $1 ! 0$ & $400 / 3400+400 / 8500$ & 1250 \\
\hline 2018 Apr 19 & 30 & Gemini + GMOS-N & $1 . " 0$ & B600 & 600 \\
\hline 2018 May 5 & 45 & Gemini + GMOS-N & $1 ! 0$ & B600 & 600 \\
\hline 2018 May 6 & 46 & DCT + DeVeny & $1 " .5$ & $300 \mathrm{~g} / \mathrm{mm}$ & 3000 \\
\hline 2018 May 10 & 50 & Keck I + LRIS & $1 . " 0$ & $600 / 4000+400 / 8500$ & $\cdots$ \\
\hline 2018 May 13 & 53 & $\mathrm{DCT}+$ DeVeny & $1 " ! 5$ & $300 \mathrm{~g} / \mathrm{mm}$ & 1800 \\
\hline 2018 May 19 & 58 & DCT + DeVeny & $1 . " 5$ & $300 \mathrm{~g} / \mathrm{mm}$ & 900 \\
\hline 2018 Sep 14 & 169 & Keck I + LRIS & $1 \stackrel{\prime \prime}{.}$ & $600 / 4000+400 / 8500$ & 1200 \\
\hline 2018 Dec 8 & 248 & Keck I + LRIS & $1 . " 0$ & $600 / 4000+400 / 8500$ & 3600 \\
\hline
\end{tabular}

Note. The Keck Low-Resolution Imaging Spectrometer (Oke et al. 1995).

(NUV) and FUV MAMA detectors, the G140L and G230L gratings were used in order to cover the spectral ranges of $1570-3180$ and $1150-1730 \AA$ at resolutions of 1.2 and $3.2 \AA$, respectively. During each visit, the observation was obtained over three $H S T$ orbits, with three equal exposures of $674 \mathrm{~s}$ in the NUV and six equal exposures of $920 \mathrm{~s}$ in the FUV. We combined the one-dimensional spectra for each epoch using inverse-variance weighting. The HST spectra of AT2018zr have similar $\mathrm{S} / \mathrm{Ns}$ to previous $H S T$ spectra for TDEs. For the FUV side of the spectrum, the $\mathrm{S} / \mathrm{N}$ is $\sim 6$, while the $\mathrm{S} / \mathrm{N}$ is $\sim 9$ for the NUV side.

\subsection{Optical Spectra}

We obtained 15 optical spectra of AT2018zr in total. The observing configuration for each spectrum is detailed in Table 1. The data obtained with the spectral energy distribution machine (SEDm), an integral field unit spectrograph, were automatically processed by the data reduction pipeline and flux-calibrated with the observations of spectrophotometric standard stars (Blagorodnova et al. 2018).

Spectra obtained with the Auxiliary-port CAMera (ACAM) on the $4.2 \mathrm{~m}$ William Herschel Telescope (WHT), the DeVeny spectrograph on the Discovery Channel Telescope (DCT), the Double Beam Spectrograph on the Palomar 200 inch (P200) telescope, and with Gemini/GMOS-N were reduced with standard IRAF routines. We performed bias subtraction and flat-fielding in the raw science frames and extracted the onedimensional spectrum. Afterward, we performed wavelength and flux calibration using observations of the arc lamp and flux standard stars from the same night.

Data obtained with the Keck Low-Resolution Imaging Spectrometer (LRIS; Oke et al. 1995) were reduced with the LRIS automated reduction pipeline. ${ }^{15}$

We measured a redshift of $z=0.071 \pm 0.001$ using $\mathrm{Ca} \mathrm{H}+\mathrm{K} \lambda \lambda 3969$, 3934 and the near-infrared Ca II triplet $(8498,8542$, and $8662 \AA)$ absorption lines that originated from the host galaxy from the late-time Keck spectrum obtained in December 2018. Our redshift measurement is consistent with

\footnotetext{
15 http://www.astro.caltech.edu/ dperley/programs/lpipe.html
}

the value $(z=0.074)$ estimated by Holoien et al. (2018) based on $\mathrm{H} \alpha$ emission.

\section{Analysis}

The interpretation of the UV and optical spectra is complicated by the presence of a high-velocity transient BAL system at $v \approx 1.55 \times 10^{4} \mathrm{~km} \mathrm{~s}^{-1}$ (Section 4.1). This BAL system manifests in UV and optical absorption troughs on top of the TDE continuum or, sometimes, the emission lines. The rest-frame optical BALs such as the hydrogen Balmer series and the metastable helium lines are particularly rare even in BALQSOs. In the following subsections, we carefully account for these absorption features while identifying and measuring the UV and optical emission lines.

\subsection{Absorption Lines}

We identified highly blueshifted hydrogen Balmer series, $\mathrm{He} \mathrm{I}^{*} \lambda 3889$, and $\mathrm{He} \mathrm{I}^{*} \lambda 5876$ transitions in the Keck optical spectrum from $\Delta t=50$ days. These features can be easily seen when the spectrum is normalized with respect to the continuum, as shown in Figure 1. We modeled these lines with a single Gaussian and present the equivalent width (EW) and FWHM measurements for the hydrogen and helium absorption lines in Table 2. Because the $\mathrm{H}$ Balmer and He I absorption lines are often accompanied by contamination from nearby features, the definition of the local continuum is more uncertain. For example, the EW measured for the $\mathrm{H} \alpha$ absorption should be viewed as a lower limit, since the blueshifted $\mathrm{H} \alpha$ absorption is close to the broad blue wing of the $\mathrm{H} \alpha$ emission. Given that the blueshifted $\mathrm{H} \gamma$ absorption may be susceptible to $\mathrm{H} \delta$ emissions in the TDE rest frame, the measured EW should also be considered as a lower limit. We measured an FWHM of $2720 \pm 200 \mathrm{~km} \mathrm{~s}^{-1}$ for $\mathrm{H} \beta$ absorption, which is the strongest blueshifted optical absorption line that is free from contamination. From these lines, we derived a mean velocity of $(15,500 \pm 400) \mathrm{km} \mathrm{s}^{-1}$ by employing the relativistic Doppler equation. Absorptions at this velocity also match with the troughs seen in the HST STIS spectra in both high (NV $\lambda 1240$, Si IV $\lambda 1397$, N IV] $\lambda 1486$, C IV $\lambda 1549, \mathrm{~N}$ III] $\lambda 1750, \mathrm{C}$ III] $\lambda 1909$, and Al III $\lambda 1857)$ and low (Si II $\lambda 1265, \mathrm{C}_{\text {II }} \lambda 1335$, [O I] $\lambda 1304$, and $\mathrm{Mg}$ II $\lambda \lambda 2796$, 2803) ionization lines in the last two epochs (days 59 and 62). 

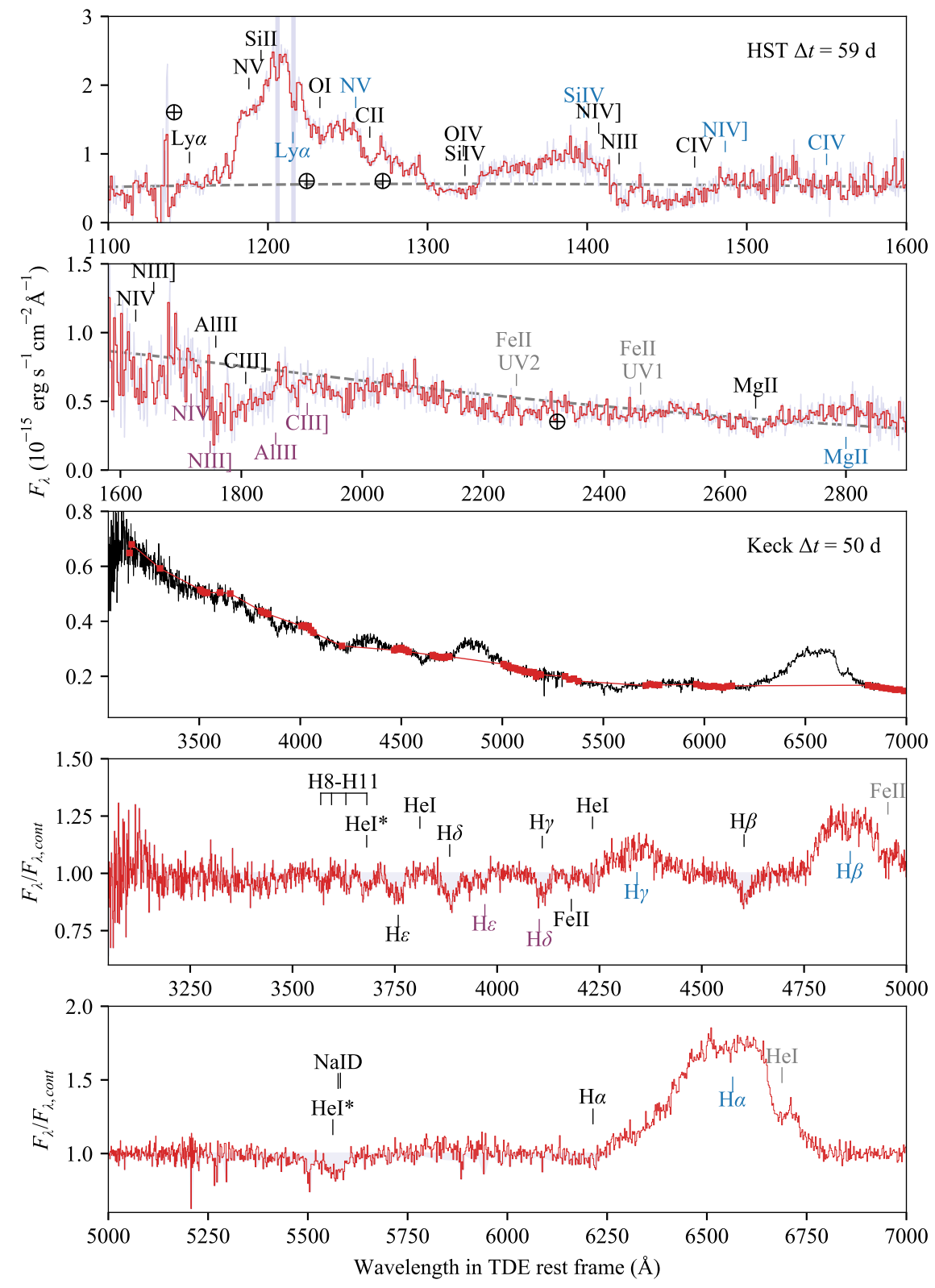

Figure 1. Zoom-in views of the UV and optical spectra with high-velocity $\left(v=15,500 \mathrm{~km} \mathrm{~s}^{-1}\right)$ absorption features indicated by black (detected) and gray (not detected) labels. The top two panels show the HST spectrum observed at $\Delta t=59$ days. Since it is hard to cleanly define the continuum in the UV, we draw a $T=22,000 \mathrm{~K}$ blackbody spectrum (gray dashed line) to guide the eye. The wavelengths of the geocoronal airglow lines are indicated by $\oplus$. Contamination from the host galaxy ( $\mathrm{Si}$ III $\lambda 1206$ and $\mathrm{Ly} \alpha$ ) is shown by the vertical gray bands. We also mark emission lines at the rest wavelength of the TDE in blue (detected) and purple (not detected). The middle panel shows the host-subtracted Keck spectrum from $\Delta t=50$ days, which has the widest wavelength coverage in the optical. We define the continuum by manually selecting the line-free regions, as marked by the red squares, and interpolating linearly between these regions. The normalized spectrum is shown in the two bottom panels.

We stacked the two HST spectra, as there is little spectroscopic evolution during this time, and we give a list of detected UV absorption lines in Table 3. We also include measurements of the absorption central wavelength and the width in the TDE rest frame, where possible.

The relative line intensities of multiple hydrogen Balmer transitions are often used to constrain neutral hydrogen column density. Assuming all photons irradiated by the continuum pass through the same amount of gas at a given velocity, the observed radiation should have the form

$$
I_{\lambda}=I_{c} e^{-\tau_{\lambda}}+B_{\lambda}\left(T_{l}\right)\left(1-e^{-\tau_{\lambda}}\right),
$$

where $I_{c}$ is the radiation of the continuum source and $T_{l}$ is the line excitation temperature. Assuming that $T_{l}$ is negligible compared to the continuum temperature $\left(T_{c}\right)$, the relative line depression can be expressed as

$$
I(v)=\frac{I_{c}-I_{\lambda}}{I_{c}}=C(v)\left(1-e^{-\tau(v)}\right)
$$

where $I(v)$ is the normalized intensity of the absorption trough, $C(\lambda)$ is the line-of-sight covering factor of the absorber in percentage, and $\tau(v)$ is the optical depth of the given transition. We note that in the case where the above assumption is invalid 
Table 2

Optical Absorption Features

\begin{tabular}{|c|c|c|c|c|c|}
\hline Line & $\begin{array}{l}\lambda_{0} \\
(\AA)\end{array}$ & $f^{\mathrm{a}}$ & $\begin{array}{c}v_{\text {rel }} \\
\left(\mathrm{km} \mathrm{s}^{-1}\right)\end{array}$ & $\begin{array}{l}\text { EW } \\
(\AA)\end{array}$ & $\begin{array}{c}\text { FWHM } \\
\left(10^{3} \mathrm{~km} \mathrm{~s}^{-1}\right)\end{array}$ \\
\hline $\mathrm{H} \alpha$ & 6564.64 & 0.641080 & $-16,560 \pm 560$ & $2.77 \pm 1.53^{b}$ & $2.52 \pm 1.32$ \\
\hline He I & 5875.00 & 0.610230 & $-15,230 \pm 120$ & $9.10 \pm 1.07$ & $3.12 \pm 0.21$ \\
\hline $\mathrm{H} \beta$ & 4862.70 & 0.119380 & $-15,380 \pm 90$ & $5.00 \pm 0.39$ & $2.72 \pm 0.20$ \\
\hline $\mathrm{H} \gamma$ & 4341.69 & 0.044694 & $-15,530 \pm 120$ & $3.55 \pm 0.86^{\mathrm{b}}$ & $2.32 \pm 0.25$ \\
\hline $\mathrm{H} \delta$ & 4102.90 & 0.022105 & $-15,390 \pm 80$ & $5.16 \pm 0.13^{\mathrm{b}}$ & $2.98 \pm 0.20$ \\
\hline $\mathrm{H} \epsilon$ & 3970.00 & 0.012711 & $-15,870 \pm 120$ & $4.11 \pm 0.16$ & $3.01 \pm 0.29$ \\
\hline $\mathrm{He} \mathrm{I}^{*}$ & 3888.65 & 0.064474 & $-15,640 \pm 160$ & $2.44 \pm 0.14$ & $2.32 \pm 0.35$ \\
\hline
\end{tabular}

Notes. EW measured in TDE rest frame.

a Oscillator strength (Wiese \& Fuhr 2009).

${ }^{\mathrm{b}}$ Measurements are subject to contamination from nearby spectroscopic features.

Table 3

Detected UV Absorption Lines in AT2018zr

\begin{tabular}{lcccc}
\hline \hline Line & $\begin{array}{c}\lambda_{0} \\
(\AA)\end{array}$ & $\begin{array}{c}\lambda_{c}^{\mathrm{a}} \\
(\AA)\end{array}$ & $\begin{array}{c}\text { Velocity } \\
\left(\mathrm{km} \mathrm{s}^{-1}\right)\end{array}$ & $\begin{array}{c}\text { FWHM } \\
\left(\mathrm{km} \mathrm{s}^{-1}\right)\end{array}$ \\
\hline N V & 1240 & $1182-1203^{\mathrm{b}}$ & $\ldots$ & $\ldots$ \\
Si II & 1263 & $1182-1203^{\mathrm{b}}$ & $\ldots$ & $\ldots$ \\
O I & 1302 & 1230 & 16,900 & 3300 \\
C II & 1334.43 & 1263 & 16,500 & 2000 \\
Si IV & 1398.0 & 1320 & 18,000 & 9800 \\
N III & 1500.0 & 1422 & 16,100 & 1800 \\
C IV & 1548.20 & 1460 & 19,000 & 8800 \\
Al III & 1856.76 & 1760 & 16,500 & 1900 \\
C III] & 1909.0 & 1810 & 16,000 & 11,000 \\
Mg II & $2796.3,2803.4$ & 2652 & 16,200 & 2600 \\
\hline
\end{tabular}

Notes.

a Central wavelength of the transition measured in host rest frame.

${ }^{\mathrm{b}}$ Cannot be determined due to blending with neighboring lines.

( $T_{l}$ is nonnegligible), the EW of the line will be altered by a factor of $\left(1-B_{\lambda}\left(T_{l}\right) / I_{c}\right)$. This effect on the observed line EW is degenerate with that of the covering factor.

The column density $N$ of an ionic species can be expressed as

$$
\begin{aligned}
N & =\frac{m_{e} c}{\pi e^{2} f \lambda_{0}} \int \tau(v) d v \\
& =\frac{3.768 \times 10^{14} \mathrm{~cm}^{-2}}{f \lambda_{0}} \int \tau(v) d v
\end{aligned}
$$

where $f$ is the oscillator strength and $\lambda_{0}$ is the rest wavelength in $\AA$. The optical depth ratio can be derived under the assumption that the absorbing gas is in local thermodynamic equilibrium. The relative line center opacity $(\tau)$ between transitions 1 and 2 can be simplified to (Arav 2001)

$$
\frac{\tau_{1}}{\tau_{2}}=\frac{g_{1} f_{1} \lambda_{1}}{g_{2} f_{2} \lambda_{2}},
$$

where $g$ is the statistical weight of the lower level, $f$ is the oscillator strength, and $\lambda$ is the wavelength of the transition.

It is clear from the line ratios that the $\mathrm{H}$ Balmer absorption lines are quite saturated. If the $\mathrm{H}$ Balmer absorptions were optically thin, the EW ratio for the $\mathrm{H}$ Balmer series would be close to $\tau_{\mathrm{H} \alpha}: \tau_{\mathrm{H} \beta}: \tau_{\mathrm{H} \gamma}: \tau_{\mathrm{H} \delta}: \tau_{\mathrm{H} \epsilon} \approx 81.5: 11.2: 3.8: 1.8: 1$. However, as shown in Table 2, the observed EWs of all of the $\mathrm{H}$
Balmer lines are very similar, suggesting that these lines are largely suppressed due to nonnegligible optical depth.

From the normalized spectrum, we also noticed that the absorptions are nonblack, meaning they do not extend down to zero. This is a clear sign of partial covering, where the absorbing material does not cover the photoionizing continuum entirely.

We first model the observed Balmer absorption lines with Equation (2) by locking the relative opacity for each transition and assuming a constant covering factor $\left(C_{0}\right)$ across all hydrogen line profiles. The best-fit parameters from directly fitting the observed spectrum are $C_{0}=0.2$ and $\tau_{0, \mathrm{H} \beta}=1.5$. We convert this to an $\mathrm{HI}(n=2)$ column density of $1.5 \times 10^{15} \mathrm{~cm}^{-2}$ with Equation (2). However, the optical depth, and therefore the $\mathrm{HI}(n=2)$ column density derived from direct fitting, may underestimate the actual values. The opacity is strongly suppressed by the fitting routine, since the model absorption line shape becomes flat-topped as it saturates, while the observed absorption features are more peak-like. One possibility is that each absorption trough is comprised of multiple unresolved narrow components with different velocities and covering factors. rather than a single broad component.

Rather than fitting the line profile, we instead use the curveof-growth method to model the total opacity summed over the $\mathrm{H}$ Balmer transitions. We exclude the use of $\mathrm{H} \alpha$ and $\mathrm{H} \gamma$ in the fit due to uncertain contributions from nearby emission lines. Shown in Figure 2, our best-fit Gaussian parameter $(b)$, ionic column density $(N)$, and covering factor are $b=313 \pm$ $170 \mathrm{~km} \mathrm{~s}^{-1}, \log _{10} N_{\mathrm{HI}(n=2)}=17.6 \pm 0.9 \mathrm{~cm}^{-2}$, and $\log _{10} C=$ $-0.6 \pm 0.2$, respectively. The high opacity of the Balmer lines places the data points on the logarithmic regime of the curve, where the EW $\left(W_{\lambda}\right)$ is relatively insensitive to $N$ $\left(W_{\lambda} \propto b \sqrt{\ln (N / b)}\right)$. The large uncertainty in $b$ may be attributed to the fact that this parameter is degenerate with the covering factor. However, the absorber needs to cover at least $15 \%$ of the continuum as measured from $\mathrm{H} \beta$. Therefore, $b$ must be narrower than $\sim 500 \mathrm{~km} \mathrm{~s}^{-1}$, which translates to an FWHM of $\sim 900 \mathrm{~km} \mathrm{~s}^{-1}$. Considering these effects, the true $N_{\mathrm{HI}(n=2)}$ should be even higher, placing a lower limit for $\tau_{\mathrm{H} \beta} \gtrsim 720$.

\subsection{UV Spectroscopy}

We present five epochs of HST spectra in Figure 3, where blackbody spectra with temperatures derived from the broadband NUV and optical photometry (see van Velzen et al. 2019) 


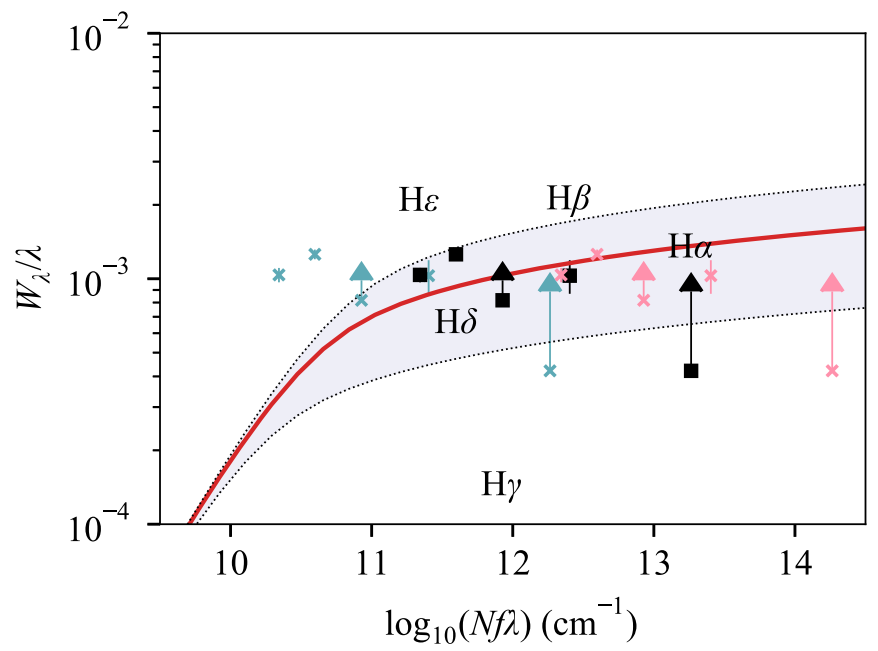

Figure 2. Curve-of-growth analysis of the Balmer absorption lines. Only $\mathrm{H} \beta$, $\mathrm{H} \delta$, and $\mathrm{H}_{\epsilon}$ are used in the fit. The best-fit curve with $b=313 \mathrm{~km} \mathrm{~s}^{-1}$, $C=0.25$, and $\log _{10} N=17.6 \mathrm{~cm}^{-2}$ is shown in red. The shaded area shows the region bounded by the $1 \sigma$ uncertainty in the Gaussian parameter $\left(\sigma_{b}=170 \mathrm{~km} \mathrm{~s}^{-1}\right)$. We also plot our data with $\log _{10} N=16.6 \mathrm{~cm}^{-2}$ (green) and $\log _{10} N=18.6 \mathrm{~cm}^{-2}$ (pink) on the growth curve. In this high-opacity regime $(\tau>1)$, the $\mathrm{EW}$ is insensitive to $N$.

are overplotted with gray dashed lines. Traditionally, observers found the UV and optical photometry measured by Swift in TDEs in agreement with a blackbody spectrum with $T \sim$ few $\times 10^{4} \mathrm{~K}$ (e.g., Holoien et al. 2016a; Hung et al. 2017). However, this agreement has not been carefully examined blueward of the Swift UVW2 band (central wavelength $1928 \AA$ ). In fact, previous work has also suggested that a significant amount of TDE radiation may be emitted in the extreme UV (e.g., Dai et al. 2018). Therefore, while the blackbody spectrum captures the general shape of the NUV spectra in all five epochs, the FUV continuum cannot be determined as accurately. We also note that this blackbody spectrum cannot account for the X-ray flux that was observed in AT2018zr. A second blackbody of $T \sim 100 \mathrm{eV}$ is required to describe the entire SED (van Velzen et al. 2019).

The UV spectra of AT2018zr show complex features, where broad emission and absorption are variable and often blended together, making it difficult to measure these lines accurately. In particular, the high-velocity BAL features (Section 4.1) only become discernible in the UV spectra in later epochs. Despite the uncertainties in FUV continuum placement, we study the evolution of the FUV lines by assuming the underlying continuum is a single blackbody, with temperatures extrapolated from NUV and optical observations. Here we summarize the observed properties of these features qualitatively.

1. The Ly $\alpha$ emission line is blueshifted by about $3000 \mathrm{~km} \mathrm{~s}^{-1}$ in all five epochs. Assuming no additional source of FUV continuum flux, the Ly $\alpha$ line flux becomes $\sim 50 \%$ more prominent after $\Delta t \approx 59$ days.

2. High-ionization N V $\lambda 1240$ emission may be present but is blended with the red wing of the broad Ly $\alpha$; therefore, the $\mathrm{N}$ V $\lambda 1240$ emission peak cannot be individually resolved.

3. The high-ionization emission line $\mathrm{Si} I V \lambda 1397$ is also blueshifted by $\sim 3000 \mathrm{~km} \mathrm{~s}^{-1}$ with respect to the rest frame of the host similar to the Ly $\alpha$ emission.

4. In the first two epochs, there is a marginally detected broad feature $\left(\mathrm{FWHM} \sim 1.3 \times 10^{4} \mathrm{~km} \mathrm{~s}^{-1}\right.$ ) at the rest wavelength of $\mathrm{Mg}$ II $\lambda \lambda 2796,2803$.
5. The likely presence of $\mathrm{NV} \lambda 1240$ emission and the absence of the C IV $\lambda 1549$ emission line in AT2018zr may be associated with the enhanced nitrogen-to-carbon $(\mathrm{N} / \mathrm{C})$ ratio (see $(\mathrm{NV} / \mathrm{C} \mathrm{IV}) \lesssim 0.1$ for QSOs; Vanden Berk et al. 2001) reported in previous TDEs ASASSN14li, iPTF16fnl, and iPTF15af (Cenko et al. 2016; Blagorodnova et al. 2019; Brown et al. 2018). We do not detect $\mathrm{N} \mathrm{IV]} \lambda 1486$ or $\mathrm{N} \mathrm{III]} \lambda 1750$ emission as in previous TDEs.

6. None of the emission peaks shifted significantly over the monitoring period, which suggests that the kinematics of the UV line-emitting region did not vary much in the first 2 months.

7. The absorption features at $v \sim 15,500 \mathrm{~km} \mathrm{~s}^{-1}$ are weak or completely absent in the first three epochs. In the last two epochs, absorption lines including high-ionization lines NV $\lambda 1240, \quad$ Si IV $\lambda 1397$, and C IV $\lambda 1549$ and lowionization lines [O I] $\lambda 1304$, C II $\lambda 1335$, Si II $\lambda 1265$, C III $\lambda$ 977, Al III $\lambda 1857$, and $\mathrm{Mg}$ II $\lambda \lambda 2796,2803$ are detected in the spectra. The UV absorption features detected in AT2018zr are consistent with those seen in low-ionization BAL (LoBAL) QSOs.

8. The UV BALs are saturated and nonblack, suggesting partial covering of the continuum source. In the later two epochs, the UV absorptions are also seen to be shallower than the emission lines (e.g., $\operatorname{Ly} \alpha$ ), which indicates that the BAL system does not cover the line-emitting region entirely, either.

\subsection{Optical Spectroscopy}

At optical wavelengths, the host galaxy contributes a nonnegligible amount of flux to the observed spectrum. Since there was no pre-flare spectrum of the host galaxy, we estimated the host flux in our spectroscopic aperture by fitting a synthetic galaxy spectrum with SDSS model magnitudes (van Velzen et al. 2019). To perform subtraction of the host flux, we first calibrated the flux level in each optical spectrum against Swift $V$-band photometry, interpolated to the spectroscopic epochs. We then convolved the synthetic host galaxy spectrum with a Gaussian kernel to account for instrumental broadening and subtracted the broadened, synthetic spectrum from our observed spectra. We show the broadened model spectrum along with the late-time Keck spectrum in Figure 4 to demonstrate the difference between the model and the data. The Keck spectrum is a superposition of the TDE continuum and lines and the host galaxy spectrum. While it is harder to assess the validity of the model with $\mathrm{H} \alpha$ and $\mathrm{H} \beta$ given the complicated structure of the emission lines from the TDE, other stellar absorption lines (such as the Ca II) that are free from TDE line emission match the model pretty well. We note that it is not possible to reconstruct all of the narrow absorption lines accurately by fitting stellar population synthesis models to broadband photometry of the host. In addition, any mismatch in the resolution between the data and model can also lead to over-/undersubtraction around the absorption line regions. We note that TDEs are dominated by low-mass black holes (Wevers et al. 2017) whose host galaxies have velocity dispersion $\left(\lesssim 100 \mathrm{~km} \mathrm{~s}^{-1}\right)$ that cannot be resolved in the lowresolution $(R<3000)$ spectra. Therefore, one should be aware that any narrow features with a width similar to the instrumental resolution appearing at the wavelengths typical 


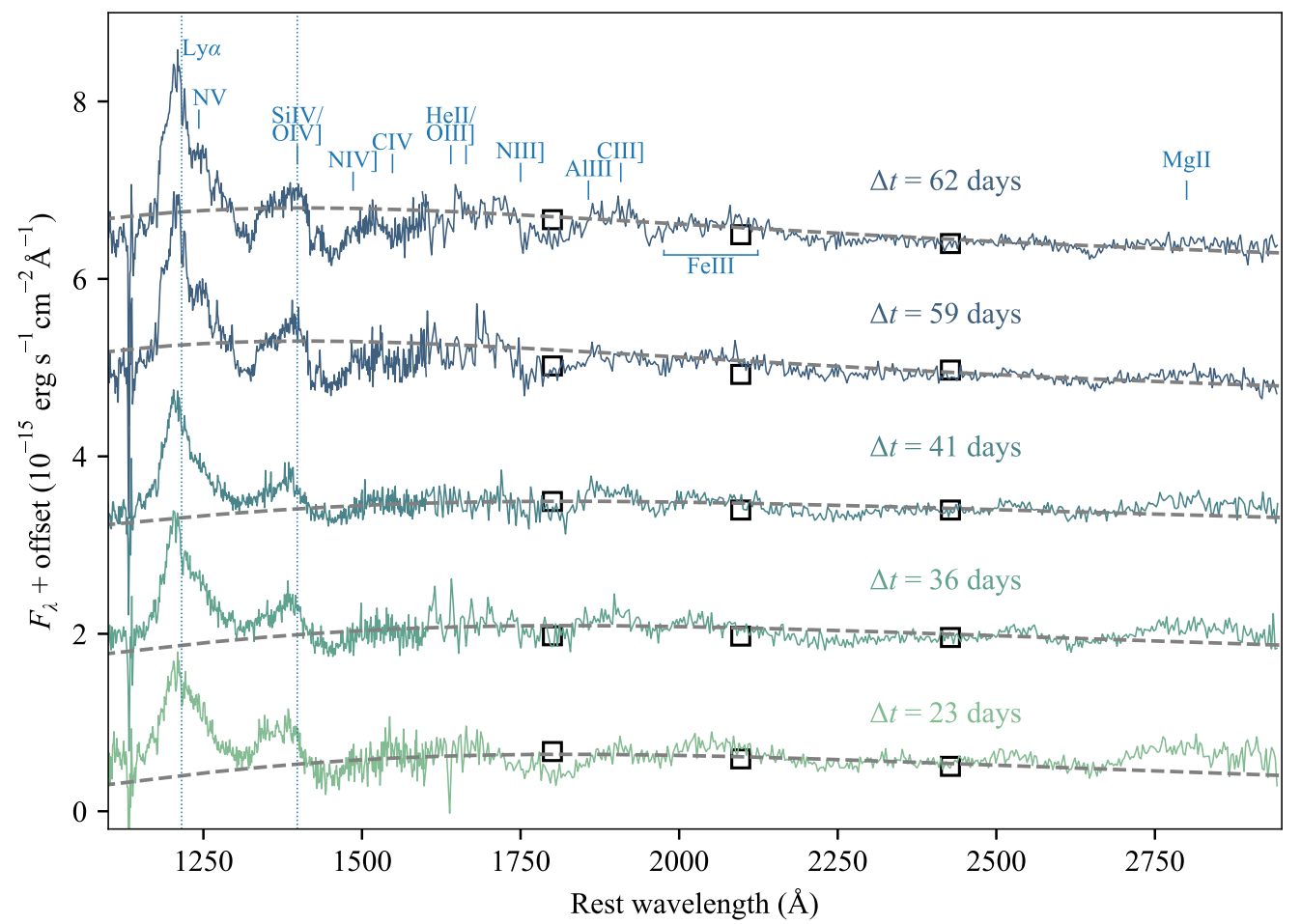

Figure 3. The HST STIS spectra of AT2018zr observed at different epochs, where $\Delta t$ indicates the rest-frame time elapsed since the peak in the $r$ band (MJD 58,194.5). To guide the eye, the gray dashed line marks the tentative FUV continuum extrapolated from a blackbody spectrum that is used to describe the NUV and optical SED. The blackbody spectra correspond to a temperature of $T=1.7 \times 10^{4} \mathrm{~K}$ for the first three epochs and $T=2.2 \times 10^{4} \mathrm{~K}$ for the last two epochs. The black squares indicate the interpolated broadband flux measured by Swift.

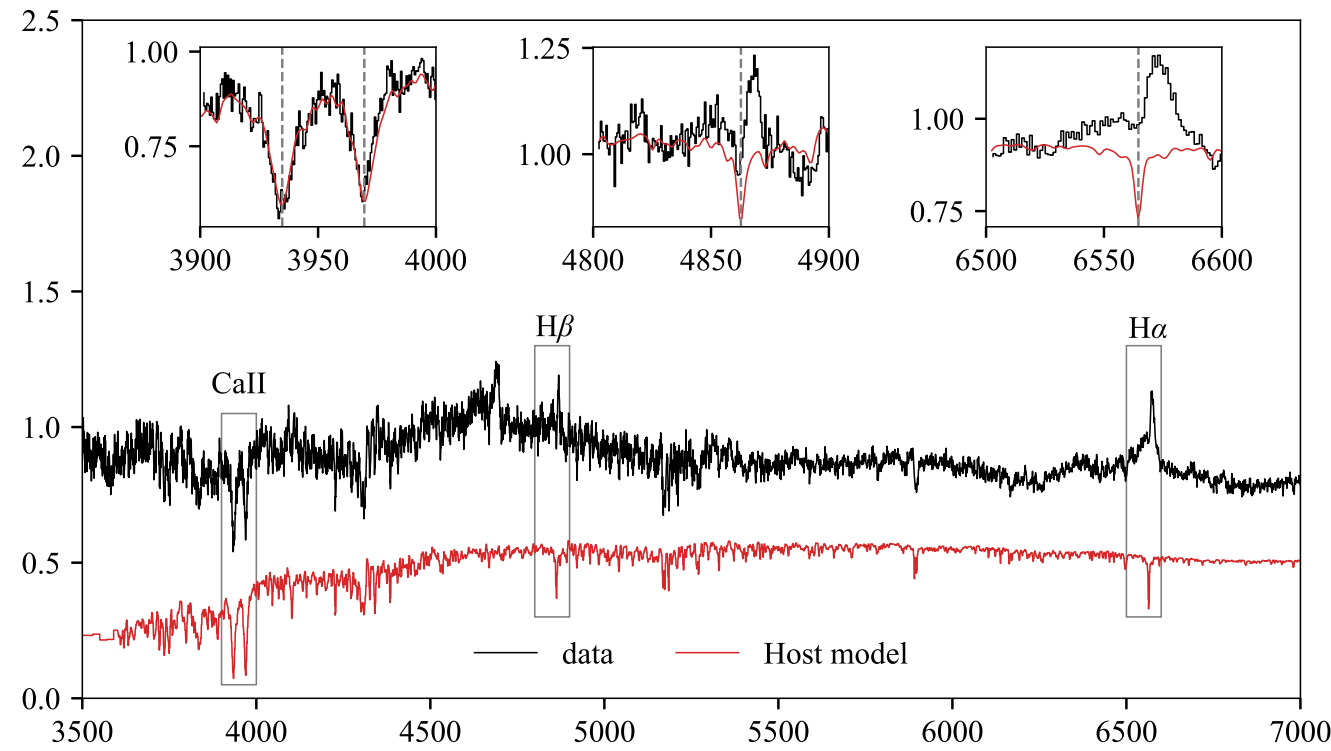

Figure 4. Comparison of the synthetic host model (red) and the late-time Keck data (black) from $\Delta t=248$. The insets show the zoom-in view of the regions around $\mathrm{Ca}$ II] $\lambda \lambda 3934,3969, \mathrm{H} \beta$, and $\mathrm{H} \alpha$. We offset the model by a constant in the insets for ease of comparison. Uncertainties in the host model or mismatched Gaussian kernel may cause over-/undersubtraction around the stellar absorption features. Such effects may leave the broad features in the final host-subtracted spectrum looking like a double-peaked profile.

of stellar absorption lines could be from the host and leave imprints on the host-subtracted spectrum. Such effects can be seen in the $\mathrm{H} \beta$ line in Figure 6, which can sometimes be misidentified as a double-peaked feature.

A montage of the host-subtracted spectra is shown in Figure 5. The fluxes are normalized to the $5500-6000 \AA$ region in rest wavelength and offset from each other for better visualization. We use the host-subtracted, Galactic extinction-corrected optical spectra in all of our analyses. The same host galaxy model is assumed throughout this work (Figure 4).

We describe our modeling of the optical emission lines before and after the Sun-constrained break separately, because the line profiles were dramatically different after the break.

Both broad $\mathrm{H} \alpha$ and $\mathrm{H} \beta$ lines were readily detected in the spectra from day 10 onward. The strength of these lines grew monotonically with time in the first 2 months. In the earlier set 


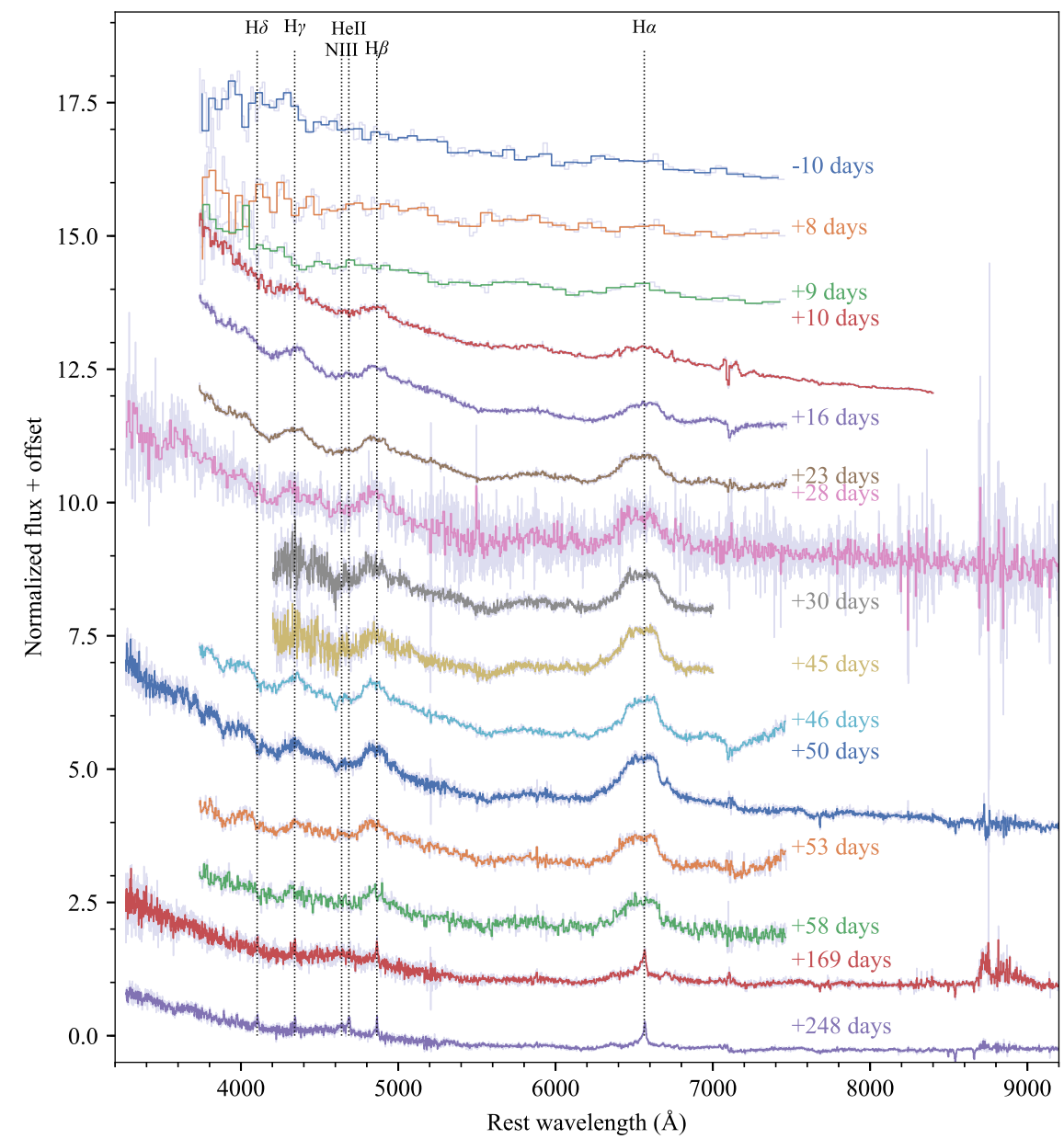

Figure 5. Host-subtracted optical spectra of AT2018zr color-coded by epoch. The first three spectra (from top) were obtained by the low-resolution SEDm. The telescope and instrument that took each spectrum are listed in Table 1. The original data are plotted in pale gray under the smoothed spectra. All spectra shown here have been binned in wavelength by 3 , except for the Keck spectrum on day 31 , which is binned by 5 pixels due to higher noise. The corresponding phase ( $\Delta t$ ) is indicated to the right of each spectrum.

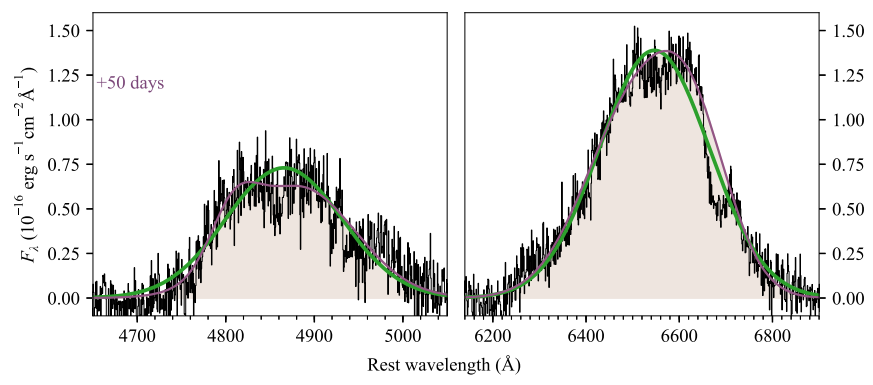

Figure 6. Gaussian model fit to $\mathrm{H} \alpha$ (right) and $\mathrm{H} \beta$ (left) lines in the Keck LRIS spectrum from 2018 May 10. We show the best-fit result of a single Gaussian (green line) and a double Gaussian model (purple line). It is clear from the plot that the flat-topped profile cannot be fitted well by a single Gaussian.

of spectra with good $\mathrm{S} / \mathrm{N}$, we noticed that the $\mathrm{H} \alpha$ and $\mathrm{H} \beta$ emission profiles were asymmetric, with what appeared to be a "dent" in the red wing (Figure 1). Although the positions of these "dents" are consistent with He I $\lambda 7065$ and Fe II $\lambda 5235$ blueshifted by the same velocity as the BAL system, the width and depth are broader and stronger than the other optical absorption lines. Given the similarity in the line profile shapes of $\mathrm{H} \alpha$ and $\mathrm{H} \beta$ (Figures 6 and 8), this asymmetry is more likely the result of emission line region geometry than contamination from neighboring absorption lines.
Interestingly, the flat-topped $\mathrm{H}$ Balmer emission profiles in AT2018zr in the earlier monitoring period differ from the Gaussian emission lines seen in other TDEs. This motivated Holoien et al. (2018) to fit these lines with a model that combines the effects of an elliptical disk, spiral arm, and wind. Here we use two different approaches to model the observed lines, including (1) phenomenological fitting with two Gaussians and (2) a radiative transfer equation in a spherical outflow.

\subsubsection{Double Gaussian Model}

In our initial attempt to fit these lines, we noticed that the line profiles, especially $\mathrm{H} \alpha$, cannot be well described by a single Gaussian (green solid line in Figure 6). Therefore, we chose to fit each flat-topped Balmer line with two Gaussians simultaneously. The choice of model was not motivated by the underlying physics of line formation but rather to obtain a satisfactory description of the line profiles. This allows us to measure the FWHM and line luminosity for comparison with other TDEs.

We first measured the continuum level in the spectral lines by performing a quadratic fit to the region enclosing the lines $(4630-5150 \AA$ for $\mathrm{H} \beta$ and $5950-6950 \AA$ for $\mathrm{H} \alpha$ ). Although we have subtracted the synthetic galaxy from our observed spectra prior to measuring the lines, we find the 
Table 4

$\mathrm{H} \alpha$ Line Parameters

\begin{tabular}{lccc}
\hline \hline $\begin{array}{l}\text { Phase } \\
\text { (days) }\end{array}$ & $\begin{array}{c}\text { FWHM } \\
\left(10^{4} \mathrm{~km} \mathrm{~s}^{-1}\right)\end{array}$ & $\begin{array}{c}\text { EW } \\
(\AA)\end{array}$ & $\begin{array}{c}\text { Luminosity } \\
\left(10^{41} \mathrm{erg} \mathrm{s}^{-1}\right)\end{array}$ \\
\hline 10 & $1.35 \pm 0.81$ & $86 \pm 17$ & $2.0 \pm 0.2$ \\
16 & $1.26 \pm 0.17$ & $89 \pm 4$ & $1.9 \pm 0.3$ \\
23 & $1.39 \pm 0.12$ & $146 \pm 4$ & $3.4 \pm 0.4$ \\
28 & $1.42 \pm 1.62$ & $188 \pm 403$ & $4.0 \pm 8.9$ \\
30 & $1.42 \pm 0.10$ & $198 \pm 3$ & $4.4 \pm 0.4$ \\
45 & $1.39 \pm 0.09$ & $224 \pm 2$ & $5.2 \pm 0.2$ \\
46 & $1.28 \pm 0.13$ & $202 \pm 7$ & $3.8 \pm 0.4$ \\
50 & $1.44 \pm 0.21$ & $265 \pm 26$ & $5.3 \pm 1.5$ \\
53 & $1.35 \pm 0.13$ & $159 \pm 4$ & $3.2 \pm 0.3$ \\
58 & $1.42 \pm 0.38$ & $190 \pm 11$ & $4.2 \pm 0.3$ \\
169 & $0.43 \pm 0.18$ & $\cdots$ & $0.2 \pm 0.1$ \\
248 & $0.35 \pm 0.07$ & $\cdots$ & $<0.1$ \\
$169^{n}$ & $0.11 \pm 0.07$ & $\cdots$ & $(8.7 \pm 2.0) \times 10^{-2}$ \\
$248^{n}$ & $0.07 \pm 0.03$ & $\cdots$ & $(4.9 \pm 0.8) \times 10^{-2}$ \\
\hline
\end{tabular}

Note. Here ${ }^{n}$ denotes the measurements for the narrow components. We do not calculate the EW in late-time spectra due to the complexity in separating the broad and narrow components.

spectra near the $\mathrm{H} \alpha$ and $\mathrm{H} \beta$ line centers to be noisy and may still have residual contribution from the host. Therefore, we masked the line centers while fitting the emission lines. In the two-component fit, we allowed the line centers, line widths, and amplitudes to vary freely and derived the best-fit model by performing $\chi^{2}$ minimization. The two best-fit Gaussians naturally center blueward and redward of the rest wavelength of the line to produce the flat-topped shape.

The best-fit two-component model is marked in purple in Figure 6. From the best-fit models, we measured the luminosity, EW, and FWHM for both $\mathrm{H} \alpha$ and $\mathrm{H} \beta$ lines. These parameters are tabulated in Tables 4 and 5 .

\subsubsection{Spherical Outflow Model for Emission Lines}

Roth \& Kasen (2018) demonstrated that in TDE outflows, the blue absorption wing of a classical P Cygni profile may appear mostly as emission if the line excitation temperature is sufficiently high. Given the similarity of their theoretical line profile to our observations, we also fit the $\mathrm{H} \alpha$ emission with this radiative transfer model proposed by Roth \& Kasen (2018) for three different epochs.

We consider a spherically symmetric, homologously expanding $(v \propto r)$ medium. A continuum photosphere is located at radius $r_{\mathrm{ph}}$, which is responsible for emitting the observed continuum flux at wavelengths near $\mathrm{H} \alpha$ at the time of interest. The gas density falls off as $r^{-2}$ at radii beyond $r_{\mathrm{ph}}$ out to a maximum radius $r_{\text {out }}$, where the velocity is $v_{\max }$. The line absorption opacity $\kappa_{l}$ and the line Doppler width $v_{D}$ are set to single values at all positions outside the continuum photosphere. The line excitation is set by $T_{\mathrm{ex}}=a+b / r$, where $a$ and $b$ are chosen so that the line excitation temperature equals a specified value $T_{\text {ex,ph }}$ at the photosphere and $T_{\text {ex,out }}$ at $r_{\text {out }}$. The Sobolev approximation can then be used to calculate the observed emission by integrating the line source function along lines of sight passing through the line-emitting material, as in Jeffery \& Branch (1990) and Roth \& Kasen (2018). The strength of the emission line with respect to the continuum flux
Table 5

$\mathrm{H} \beta$ Line Parameters

\begin{tabular}{lllc}
\hline \hline $\begin{array}{l}\text { Phase } \\
\text { (days) }\end{array}$ & $\begin{array}{c}\text { FWHM } \\
\left(10^{4} \mathrm{~km} \mathrm{~s}^{-1}\right)\end{array}$ & $\begin{array}{c}\text { EW } \\
(\AA)\end{array}$ & $\begin{array}{c}\text { Luminosity } \\
\left(10^{41} \mathrm{erg} \mathrm{s}^{-1}\right)\end{array}$ \\
\hline 10 & $1.14 \pm 0.22$ & $21 \pm 2$ & $1.0 \pm 0.2$ \\
16 & $1.05 \pm 0.56$ & $27 \pm 11$ & $1.1 \pm 0.6$ \\
23 & $1.23 \pm 0.18$ & $33 \pm 3$ & $1.2 \pm 0.2$ \\
28 & $1.17 \pm 2.78$ & $46 \pm 283$ & $1.6 \pm 5.3$ \\
30 & $1.17 \pm 0.22$ & $42 \pm 2$ & $1.4 \pm 0.2$ \\
45 & $0.96 \pm 0.63$ & $39 \pm 13$ & $1.2 \pm 0.5$ \\
46 & $0.96 \pm 0.59$ & $41 \pm 11$ & $1.3 \pm 0.5$ \\
50 & $1.20 \pm 0.09$ & $47 \pm 1$ & $1.5 \pm 0.1$ \\
53 & $1.11 \pm 0.36$ & $27 \pm 12$ & $0.9 \pm 0.4$ \\
58 & $0.89 \pm 1.04$ & $34 \pm 48$ & $1.1 \pm 0.9$ \\
169 & $0.15 \pm 0.05$ & $\cdots$ & $<0.1$ \\
248 & $0.19 \pm 0.02$ & $\cdots$ & $<0.1$ \\
$169^{n}$ & $0.05 \pm 0.02$ & $\cdots$ & $(2.0 \pm 1.4) \times 10^{-2}$ \\
$248^{n}$ & $0.04 \pm 0.01$ & $\cdots$ & $(1.8 \pm 0.4) \times 10^{-2}$ \\
\hline
\end{tabular}

Table 6

Fiducial Parameters for the Spherical Outflow Model of the $\mathrm{H} \alpha$ Line Profiles

\begin{tabular}{lccccc}
\hline \hline $\begin{array}{l}r_{\text {ph }} \\
(\mathrm{cm})\end{array}$ & $\begin{array}{c}r_{\text {out }} \\
(\mathrm{cm})\end{array}$ & $\begin{array}{c}v_{\max } \\
\left(10^{4} \mathrm{~km} \mathrm{~s}^{-1}\right)\end{array}$ & $\begin{array}{c}\rho_{\text {ph }} \\
\left(\mathrm{g} \mathrm{cm}^{-3}\right)\end{array}$ & $\begin{array}{c}T_{\text {ex,ph }} \\
(\mathrm{K})\end{array}$ & $\begin{array}{c}T_{\text {ex,out }} \\
(\mathrm{K})\end{array}$ \\
\hline $10^{15}$ & $3 \times 10^{15}$ & 1.75 & $1.16 \times 10^{-15}$ & $3 \times 10^{4}$ & $10^{4}$ \\
\hline
\end{tabular}

Note. These values were kept the same between the three spectral epoch fits. The gas density is assumed proportional to $r^{-2}$, and the gas velocity is assumed proportional to $r$ (and is entirely radially outflowing). The line excitation temperature drops as $a+b / r$, where $a$ and $b$ are chosen to match the listed excitation temperature values at the photosphere radius and the outer radius.

Table 7

Continuum Fluxes and Sobolev Depths Used in the $\mathrm{H} \alpha$ Line Profile Fits, Which Were Varied between Spectral Epochs

\begin{tabular}{lccc}
\hline \hline$\Delta t$ (days) & 23 & 30 & 50 \\
\hline$\tau_{S}\left(r_{\text {ph }}\right)$ & 0.39 & 0.48 & 0.47 \\
$\begin{array}{l}\text { Continuum flux } \\
\left(\operatorname{erg~cm}^{-2} \mathrm{~s}^{-1} \AA^{-1}\right)\end{array}$ & $1.02 \times 10^{-16}$ & $9.80 \times 10^{-17}$ & $6.81 \times 10^{-17}$ \\
\hline
\end{tabular}

is then related to the Sobolev depth $\tau_{S}$ at each radius:

$$
\tau_{S}(r)=\sqrt{\pi} \rho(r) \kappa_{l} v_{D}\left(\frac{d v}{d r}\right)^{-1} .
$$

For the conditions considered here, the electron-scattering optical depth is negligible for the line photons.

Rather than perform a full multidimensional fit, we set all parameters to the fiducial values listed in Table 6 and then adjust the value of $\tau_{S}\left(r_{\mathrm{ph}}\right)$ between spectral epochs until we judge by eye that a satisfactory fit has been achieved. The continuum flux is also adjusted between epochs to match what is observed. The resulting values are listed in Table 7. Given the definition of $\tau_{S}$, the values of $\kappa_{l}$ and $v_{D}$ are degenerate in this formulation, since only their product enters into the calculation, as long as $v_{D} \ll v_{\max }$. The results are plotted in Figure 7 .

\subsubsection{Late-time Optical Spectrum}

The spectroscopic features showed dramatic changes (Figure 8) at late times $(\Delta t=169$ and 248 days). First, He II $\lambda 4686$ and 


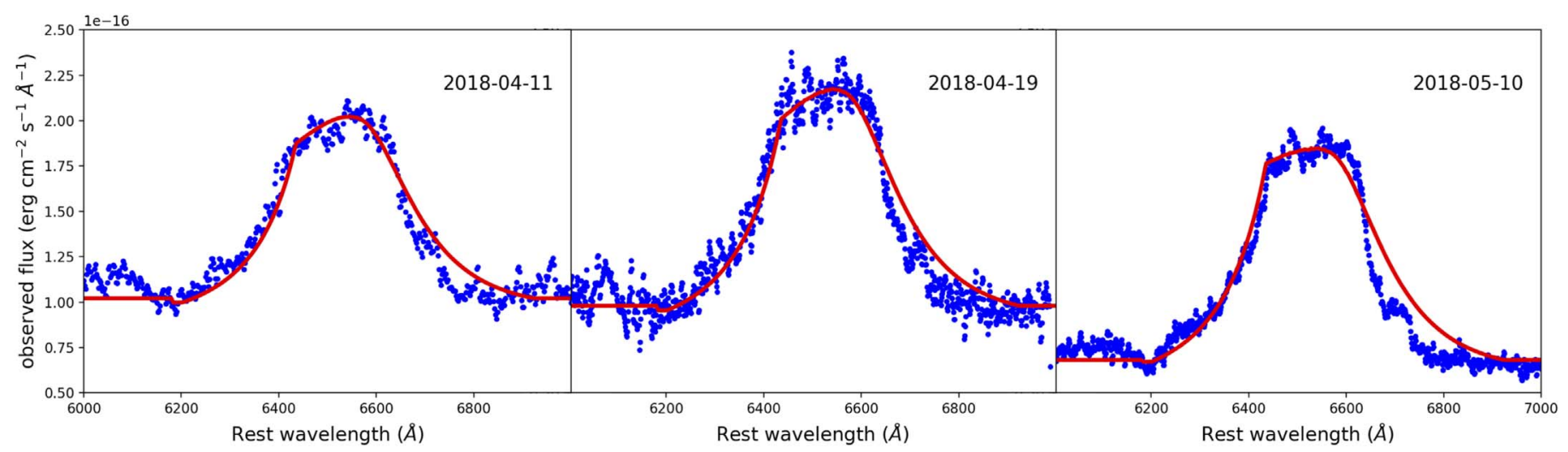

Figure 7. The $\mathrm{H} \alpha$ line profiles, with fits to a spherical outflow model for the broad emission. An additional absorption component at the highest velocities is discussed in Section 4.1.
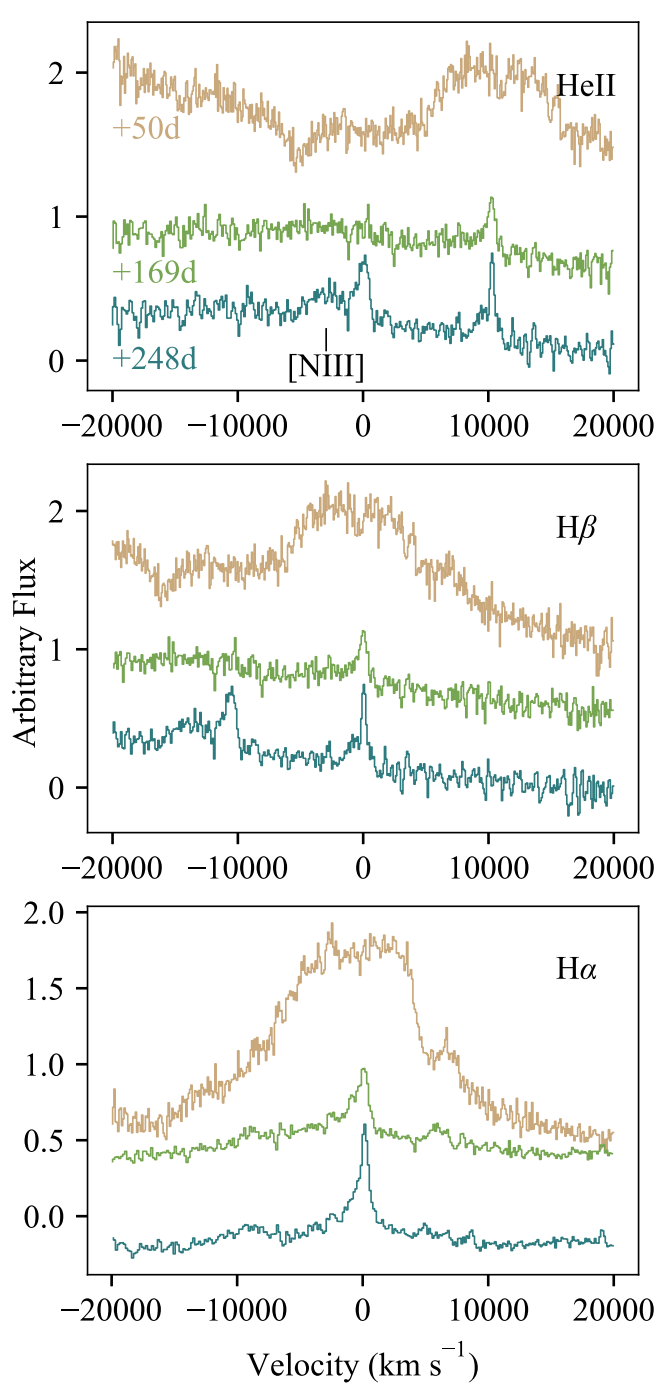

Figure 8. Velocity profiles of $\mathrm{He}$ II $\lambda 4686 \mathrm{H} \beta$ and $\mathrm{H} \alpha$ of AT2018zr at $\Delta t=50,169$, and 248 days. Narrow $\mathrm{H} \alpha, \mathrm{H} \beta$, and He II $\lambda 4686$ emission emerged 6 months after maximum light. The narrow line widths are on the order of $1000 \mathrm{~km} \mathrm{~s}^{-1}$, which is only $\sim 10 \%$ of the broad Balmer line widths from earlier epochs. We note that on day $50, \mathrm{H} \alpha$ and $\mathrm{H} \beta$ have a similar velocity profile that is dented on the red wing, which is likely to arise from the geometry of the line-forming region.
N III $\lambda 4640 \AA$, a strong transition of the Bowen fluorescence (BF) mechanism, emission lines are readily detected on day 248 , while the detections are somewhat tentative on day 169. In BF, the He II Ly $\alpha$ photons at $303.783 \AA$ can excite a number of nearby transitions, which decay into optical emission lines such as O III (at 3133, 3429, and $3444 \AA$ ) and N III $\lambda 4640 \AA$. Emission lines of $\mathrm{BF}$ have been detected in systems such as planetary nebulae and X-ray binaries and also in TDE iPTF15af (Blagorodnova et al. 2019).

The late appearance of the He II $\lambda 4686$ emission is unlike most other TDEs to date (e.g., PS1-10jh; Gezari et al. 2012), where hydrogen emission is often suppressed relative to helium at early times (Roth et al. 2016). The absence of the He II $\lambda 4686$ line may be attributed to the lack of He II ionizing photons $\left(\chi_{\text {ion }}=54.4 \mathrm{eV}\right)$ at earlier times. This is supported by the fact that the blackbody temperature of AT2018zr is cooler than many other TDEs at first but warms up drastically at later times (van Velzen et al. 2019). The hot continuum indicated by the late-time Swift observations would naturally explain the emergence of the He II $\lambda 4686$ emission.

The broad $\mathrm{H} \alpha$ and $\mathrm{H} \beta$ emission had become extremely weak when we revisited this source on $\Delta t=169$ days. Instead, narrow $\mathrm{H} \alpha$ and $\mathrm{H} \beta$ emission emerged at a velocity close to the rest frame of the host galaxy. The most striking difference is found in the $\mathrm{H} \alpha$ and $\mathrm{H} \beta$ lines, which are now dominated by two strong narrow components, whereas the broad $\mathrm{H} \alpha$ and $\mathrm{H} \beta$ emission have vastly diminished, though they can still be seen. We measured $\mathrm{H} \alpha$ and $\mathrm{H} \beta$ in the late-time spectra with a double Gaussian model, where both broad and narrow components are initially centered at the rest wavelength of the transition. We show the best-fit results in Tables 4 and 5 . The newly formed narrow $\mathrm{H} \alpha$ and $\mathrm{H} \beta$ emission has FWHMs on the order of $1000 \mathrm{kms}^{-1}$, which correspond to a virial radius of $\sim 6 \times 10^{17} \mathrm{~cm}$ assuming a black hole mass of $10^{7} \mathrm{M}_{\odot}$.

\section{Discussion}

\subsection{UV Spectra of TDEs}

The TDE AT2018zr is the fourth one optically discovered with $H S T$ UV spectroscopic observations. We compare its $H S T$ spectrum with the other three-ASASSN-14li, iPTF15af, and iPTF16fnl—in Figure 9 (Cenko et al. 2016; Blagorodnova et al. 2019; Brown et al. 2018). The only other TDE with a UV 


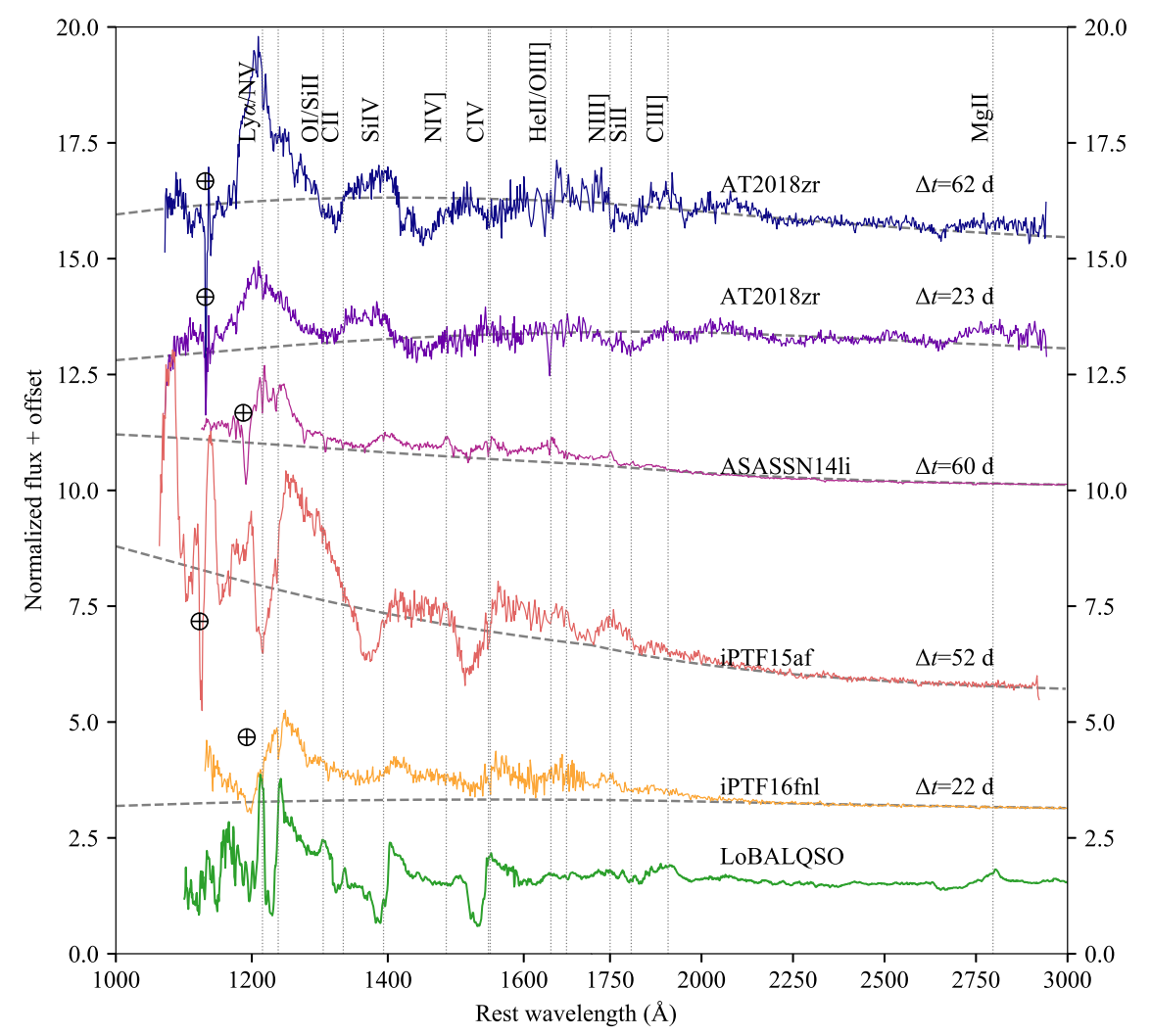

Figure 9. Comparison with the UV spectra of other TDEs. The gray dashed line at the bottom of each TDE spectrum marks the continuum, which is approximated by a blackbody spectrum of the corresponding temperature.

spectroscopic sequence (Brown et al. 2018) is iPTF16fnl. For comparison, we also show a composite spectrum of LoBAL QSOs from the FIRST Bright Quasar Survey (Brotherton et al. 2001) in green.

Because the high-velocity absorption features were weak or completely absent in the first two HST epochs, we are able to identify that the emission peaks of Ly $\alpha$ and Si IV $\lambda 1397$ are blueshifted by $\sim 3000 \mathrm{~km} \mathrm{~s}^{-1}$ with respect to the TDE rest frame. The only object that exhibit blueshifted UV emission (Ly $\alpha$, Si IV 11397) among the TDEs is AT2018zr. In ASASSN-14li, the emission lines are near the systemic host velocity (Cenko et al. 2016), while in iPTF15af and iPTF16fnl, the emission lines are systematically redshifted (Blagorodnova et al. 2019; Brown et al. 2018). The velocity of the emission lines may arise from a combination of effects, including the geometry, kinematics, and optical depth. In iPTF15af, the redshifted broad emission lines are most likely caused by the absorption of the blue wing at $v \approx-5000 \mathrm{~km} \mathrm{~s}^{-1}$. Brown et al. (2018) obtained three epochs of $H S T$ spectra of iPTF16fnl spanning a time coverage of $\sim 1$ month. While analyzing the evolution of iPTF16fnl, they found different evolution of the central wavelength for high- and low-ionization lines. Specifically, they found that the high-ionization emission lines were initially redshifted by $v \sim 2000 \mathrm{~km} \mathrm{~s}^{-1}$ but then evolved to peak near the wavelengths of the corresponding transitions at later times, while the low-ionization lines showed no apparent shift at any given time. Since the UV spectrum of iPTF16fnl exhibits blueshifted absorption troughs for Si IV $\lambda 1397$ and C IV $\lambda 1549$ (FWHM $\sim 6000 \mathrm{~km} \mathrm{~s}^{-1}$ ), they suggested that the redshifted emission peaks may be due to the blue wings of the emission lines being significantly absorbed as a result of an outflow at earlier times. This would be consistent with our finding that the BAL system is fast-evolving, on a timescale of days to weeks. On the other hand, the peak of $\operatorname{Ly} \alpha$ and Si IV $\lambda 1397$ emission in AT2018r did not show any significant shift during the $H S T$ monitoring.

It is commonly observed in QSOs that the high-ionization lines (e.g., C IV $\lambda 1549$ ) are systematically blueshifted with respect to the low-ionization lines (e.g., Gaskell 1982; Richards et al. 2002). This blueshift is often attributed to the presence of a radiatively driven wind (Gaskell 1982; Murray et al. 1995). This may also be responsible for blueshifting $(v \approx$ $3000 \mathrm{~km} \mathrm{~s}^{-1}$ ) the Si IV $\lambda 1397$ and even the Ly $\alpha$ emission in AT2018zr. In fact, the velocity profile of the strong, blueshifted Ly $\alpha$ emission is more similar to the Si IV $\lambda 1397$ emission than to the $\mathrm{H} \alpha$ emission (Figure 10). In the class of AGNs with double-peaked broad Balmer emission lines, it has been observed that the $\operatorname{Ly} \alpha$ emission tends to be single-peaked and narrower in width (Eracleous \& Halpern 2003). This difference has been attributed to Ly $\alpha$ being emitted from higher-ionization gas originating in a wind, compared to higher-density gas in the accretion disk producing the lowionization lines (Eracleous \& Halpern 2003). Based on the similar velocity, the observed $\mathrm{Ly} \alpha$ emission may naturally form in the same outflowing gas with intermediate velocity $\left(v \approx 3000 \mathrm{~km} \mathrm{~s}^{-1}\right)$ as the Si IV $\lambda 1397$ emission. We measured a Ly $\alpha$ luminosity of $5.08 \times 10^{43} \mathrm{erg} \mathrm{s}^{-1}$ from the first $H S T$ epoch, which is at least 2 orders of magnitude stronger than the $\mathrm{H} \alpha$ emission observed on the same night and $\gtrsim 10$ times larger than the expected value from case $\mathrm{B}$ recombination $(\approx 8.7)$. This could be a consequence of continuum fluorescence in the Lyman lines (Gaskell 2017). 


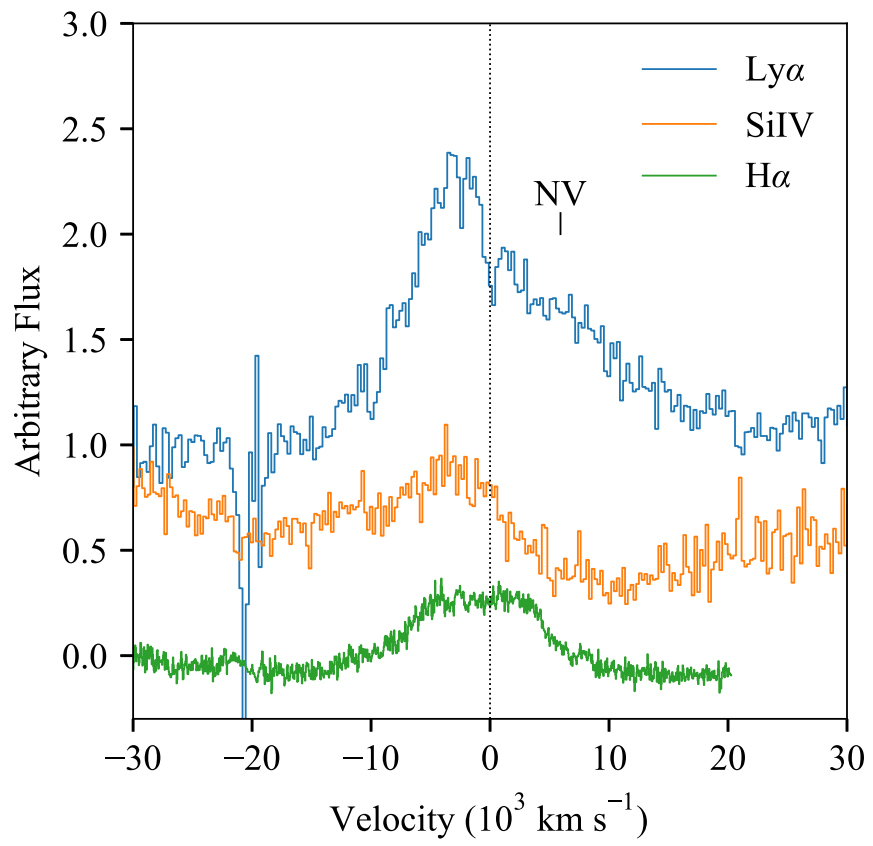

Figure 10. Velocity profiles of the $\operatorname{Ly} \alpha$, Si IV $\lambda 1397$, and $\mathrm{H} \alpha$ emission lines at $\Delta t=36$ days. Both Ly $\alpha$ and Si IV $\lambda 1397$ show a systemic blueshift of $\sim 3000 \mathrm{~km} \mathrm{~s}^{-1}$ relative to $\mathrm{H} \alpha$, which suggests the lines were formed in locations with different kinematics.

\subsection{Broad Balmer Emission Lines}

In Figure 11, we show the evolution of the FWHM of $\mathrm{H} \alpha$ and $\mathrm{H} \beta$ measured with the best-fit double Gaussian model. The $\mathrm{H} \alpha$ line width of AT2018zr is comparable to ASASSN-14ae at earlier times but broader than that of the other TDEs by a factor of $\sim 30 \%$. The FWHMs of the broad $\mathrm{H} \alpha$ and $\mathrm{H} \beta$ components show little evolution before the 3 month long observational gap but decreased by a factor of $\gtrsim 3$ at late time. This long-term trend of line narrowing is also seen in other TDEs (Figure 11), such as ASASSN-14ae and ASASSN-14li (Holoien et al. 2014, 2016b), though the blackbody luminosity in AT2018zr did not decrease monotonically like in the other TDEs (van Velzen et al. 2019). As derived by van Velzen et al. (2019), the blackbody luminosity of AT2018zr decreased with time until $\Delta t_{\text {peak }} \sim 70$ days post-peak and remained nearly constant up to $\Delta t_{\text {peak }} \approx 250$ days. Nevertheless, neither the line-width evolution in AT2018zr nor that in other TDEs conforms with the results of AGN reverberation mapping, where the $\mathrm{H} \alpha$ line width increases as the luminosity decreases due to less recombination at outer radii (Holoien et al. 2016b), hence the absence of the low velocity dispersion components.

The earlier $(\Delta t \leqslant 58)$ broad $\mathrm{H} \alpha$ FWHM corresponds to a virial radius of $\approx 1.5 \times 10^{15} \mathrm{~cm}$, assuming a black hole mass of $10^{6.9} M_{\odot}$ (van Velzen et al. 2019). During this period, the broad $\mathrm{H} \alpha / \mathrm{H} \beta$ line ratio follows the case $\mathrm{B}$ value closely, with a mean of 3.1 and a standard deviation of 0.8 . At late time, the average $\mathrm{H} \alpha / \mathrm{H} \beta$ line ratio is $7.1 \pm 0.9$. The measured narrow $\mathrm{He}$ II $\lambda 4686 / \mathrm{H} \alpha$ ratio from the last spectrum ( $\Delta t=248$ days) is $\approx 0.4$, which is consistent with the case $\mathrm{B}$ prediction assuming solar abundance (Hung et al. 2017). The presence of strong He II $\lambda 4686$ emission implies a strong ionizing continuum beyond $54 \mathrm{eV}$ and gas with a high ionization parameter (Gaskell \& Rojas Lobos 2014).

The flat-topped line profile in AT2018zr is unique among the optical TDEs discovered so far. In AGNs, the flat-topped line

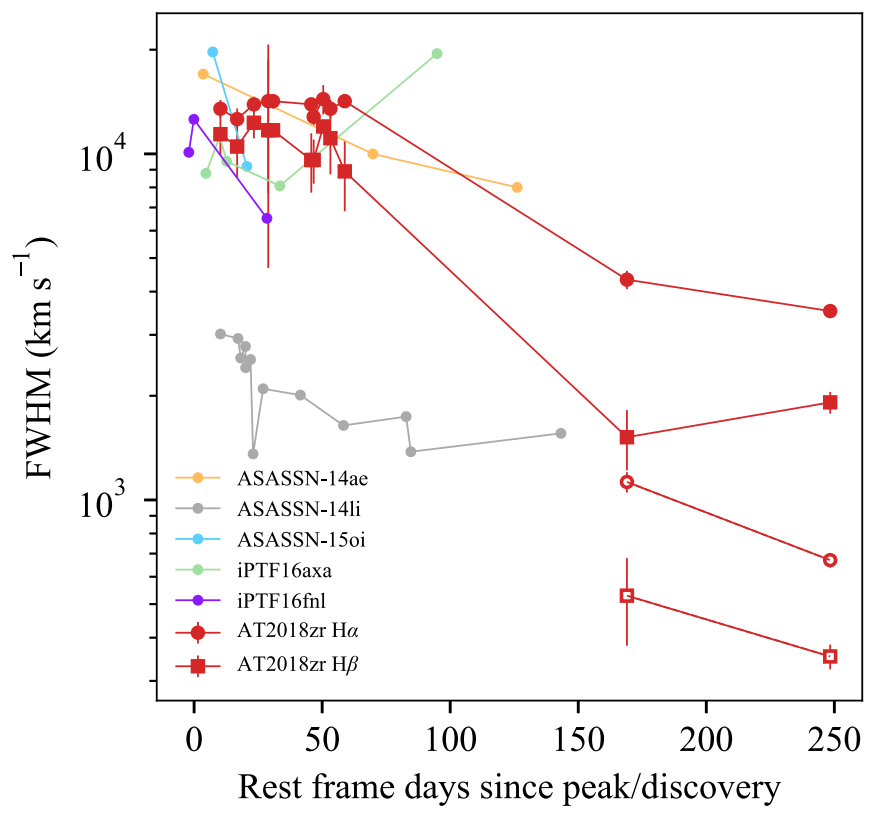

Figure 11. Evolution of the FWHM of $\mathrm{H} \alpha$ (red circles) and $\mathrm{H} \beta$ (red squares) for AT2018zr compared with the FWHM evolution of $\mathrm{H} \alpha$ for other wellstudied TDEs. The $\mathrm{H} \alpha$ and $\mathrm{H} \beta$ line widths declined much slower at early times than the other TDEs. The late-time line widths are almost as narrow as ASASSN-14li. The FWHMs of the narrow components are shown with open symbols.

shape is often attributed to the orbital motion of a Keplerian disk. Double-peaked emission lines have been observed in many AGNs, which are believed to originate from the outer part of an accretion disk at $\approx 1000 r_{g}$ (e.g., Chen et al. 1989; Eracleous \& Halpern 1994; Strateva et al. 2003; Gaskell 2009) illuminated by a central ionizing source. In addition to Doppler broadening, relativistic effects are incorporated to model the asymmetries seen in the double-peaked emission lines. For example, a circular relativistic disk model (Chen et al. 1989; Mathews 1982) is often employed to explain AGN spectra where the emission lines have a double-peaked shape with a stronger blue peak. The opposite case, in which the red peak is stronger, could be achieved with an elliptical disk (Eracleous et al. 1995). Such a model has been employed in fitting TDE lines in several cases, for example, PTF09djl (Liu et al. 2017) and ASASSN-14li (Cao et al. 2018).

Motivated by the theoretical line profiles of an elliptical disk, Holoien et al. (2018) modeled the $\mathrm{H} \alpha$ line of AT2018zr (PS18kh) at different epochs with the combining effects of an elliptical disk, a spiral arm, and wind. Their model provides a reasonable fit to the observed $\mathrm{H} \alpha$ emission line shape. However, we find very little evidence for the double-peaked $\mathrm{H} \alpha$ line profile as claimed by Holoien et al. (2018) in our hostsubtracted spectra. We suspect that the dip around line center in their data is most likely due to the host starlight, which may not have been cleanly removed. As shown by their fit, the observed line center flux always drops more steeply than what the elliptical disk model can reproduce. Furthermore, for a pure Keplerian disk, a flat-top line is only expected under very specific conditions, i.e., when the disk rotation axis is $\lesssim 15^{\circ}$ (Landt et al. 2014).

In addition, the X-ray observations favor the presence of an accretion disk (van Velzen et al. 2019), which is not expected in the elliptical disk model. In the case where the debris streams 
do not circularize efficiently, the streams may retain high eccentricities without forming a standard accretion disk (Svirski et al. 2017). In this model, streams may plunge directly into the black hole when some of the angular momentum is removed, without losing any energy. Lastly, the outer radius of the elliptical disk derived by Holoien et al. (2018) is on the order of $15,000 r_{g}$, which is almost 2 orders of magnitude greater than the self-intersection radius of a nonspinning black hole with $M_{\mathrm{BH}}=10^{6.9} M_{\odot}$ (e.g., Wevers et al. 2017; Dai et al 2015).

Overall, we do not find it necessary to invoke the elliptical disk model to interpret the spectral line shape. Our spherical outflow model provides a natural explanation for the flattopped line shape. Interestingly, the maximum outflow velocity $v_{\max } \sim 1.75 \times 10^{4} \mathrm{~km} \mathrm{~s}^{-1}$ employed by our model is consistent with the velocity of the BAL system. This coincidence may be explained if the absorption lines are produced by this spherically expanding material at its outer edge when it scatters photons outside our line of sight.

\subsection{High-velocity Transient BAL System}

Observations of AT2018zr indicate the presence of the first transient LoBAL system in a TDE, in which both high- and low-ionization absorption lines are present. In addition, this transient LoBAL system contains intrinsic hydrogen Balmer, $\mathrm{He} \mathrm{I}^{*} \lambda 3889$, and He I ${ }^{*} \lambda 5876$ absorption features. It turns out that such systems are very rare, even in QSOs. Blueshifted, broad high-ionization absorption lines are seen in about $10 \%-$ $20 \%$ of the optically selected QSOs (Hewett \& Foltz 2003; Reichard et al. 2003; Trump et al. 2006; Ganguly et al. 2007) and are often attributed to accretion disk outflows. However, only about $15 \%$ of the BALQSOs also show LoBALs such as Mg II $\lambda \lambda 2796$, 2803. A small fraction (15\%) of the LoBAL QSOs that show Fe II or Fe III absorption, such as that in Mrk 231, are dubbed FeLoBALs (e.g., Veilleux et al. 2016). Since AT2018zr lacks common Fe II absorption in the NUV, it does not fit into the FeLoBAL category. Currently, there are only a handful of BALQSOs that exhibit hydrogen Balmer absorption lines (e.g., Aoki 2010; Ji et al. 2012; Zhang et al. 2015; Shi et al. 2016; Sun et al. 2017; Schulze et al. 2018).

Although stellar Balmer absorption lines are often seen in galaxy spectra, it is unlikely that the ones we see in AT2018zr are due to intervening galaxies or clouds for the following reasons. First, high-ionization absorption troughs are detected at the same velocities as the $\mathrm{H}$ Balmer absorption lines, suggesting a dynamical association between them. Second, multi-epoch optical spectra captured the appearance and disappearance of the $\mathrm{H}$ Balmer absorptions on a timescale of days, which is unexpectedly short if the absorption is due to intervening gas. Third, the He I ${ }^{*} \lambda 3889$ line must arise from the metastable $2^{3} \mathrm{~S}$ level, which is mainly populated by a recombination of singly ionized helium ions that require a significant amount of photons with $E>24.6 \mathrm{eV}$ (Rudy et al. 1985). The He I ${ }^{*} \lambda 3889$ is not seen in the interstellar medium, since the diffuse stellar background offers too few photons that can ionize helium and too many with $E>4.8 \mathrm{eV}$ that can ionize the electron in its metastable state. Lastly, since the TDE is relatively nearby, we should be able to resolve the intervening galaxies with imaging, yet we do not find any.

\subsubsection{Outflows}

Following a star's disruption, the mass initially falls back at a super-Eddington rate and gradually decreases below the Eddington rate. Super-Eddington accretion is capable of driving powerful outflows with radiation pressure (e.g., King \& Pounds 2003; Ohsuga et al. 2005). Strubbe \& Quataert (2011) made the first predictions of the spectroscopic signatures of super-Eddington outflows in a TDE environment. Their predicted spectrum is characterized by broad, blueshifted ( $v_{\text {wind }} \sim 0.01 c-0.1 c$ ) absorption features in the super-Eddington phase. Most of these absorption features are high-ionization lines $\left(\chi_{\text {ion }}>13.6 \mathrm{eV}\right)$ because of the assumption of a hot continuum with a temperature $T_{\mathrm{bb}} \gtrsim 10^{5} \mathrm{~K}$. Since the velocity and density of the outflow are viewing angle-dependent, their model is also able to produce spectra with more NUV and optical absorption lines, such as Mg II $\lambda \lambda 2796,2803$ and the hydrogen Balmer lines, as a result of a softer continuum. In general, their model predicts a spectrum similar to that of a BALQSO and hence is similar to our observations of AT2018zr. However, they predicted a rapid photospheric temperature evolution and a steep $t^{-95 / 36}$ (Strubbe \& Quataert 2009; Lodato \& Rossi 2011) decline in optical flux, which is not seen in the data.

More recent work has refined the theoretical understanding of how TDEs might be associated with wide-angle outflows. Miller (2015) suggested that winds launched by radiation pressure on absorption lines may remove material as it is drawn in through an extended disk, which would lead to a range of outflow velocities. Metzger \& Stone (2016) studied massloaded winds launched by both accretion luminosity and energy released during debris circularization. They found minimum outflow velocities of $\sim 10,000 \mathrm{~km} \mathrm{~s}^{-1}$, similar to the velocities measured here. They also pointed out that a shell of promptly launched material will surround the wind consisting of material that has fallen back more recently. Colder material at the edge of this shell might provide the origin of the blueshifted absorption troughs seen in the optical spectra of this event.

Another approach has been to perform hydrodynamic simulations of super-Eddington accretion flows, accounting for the effects of radiation pressure, magnetic fields, and general relativity, and apply these findings to TDEs (Sa̧dowski et al. 2014; Jiang et al. 2017; Dai et al. 2018; Curd \& Narayan 2019). In particular, Dai et al. (2018) emphasized the viewing angle dependence of the outflow and its resulting emission. While material ejected in the polar direction has a relativistic velocity and is transparent to X-rays, material launched closer to the midplane has a slower velocity due to mass loading, and X-ray emission is highly suppressed along those lines of sight. The results for such a viewing geometry are consistent with many aspects of this event, although the timedependent behavior of this model requires further study.

The simplest super-Eddington outflow models assume that the wind velocity is a near-unity fraction of the escape velocity of the gas from the position where it is launched (Strubbe \& Quataert 2009; Lodato \& Rossi 2011). This would require the outflowing gas to be launched from a radius of $\approx 10^{15} \mathrm{~cm}$ to match the observed velocity of $0.05 c$ for the absorption features, though the black hole mass estimation may be somewhat uncertain. However, this radius derived from the velocity of the absorption features appears to be in tension with the blackbody radius derived from the UV and optical 
photometry. The blackbody radius is found to be steadily decreasing from $10^{15.1} \mathrm{~cm}$ near peak to $\lesssim 10^{14} \mathrm{~cm}$ at $\Delta t \sim 250$ days (van Velzen et al. 2019). In this picture, the outflow would be launched at a radius slightly larger than that traced by the blackbody radius in AT2018zr when we first observed the $\mathrm{H} \beta$ absorption on day 30. Meanwhile, our modeling of the emission lines requires the continuum photosphere to be located within the edge of the outflow. Accounting for deviations from a pure blackbody emitter as appropriate for a scattering-dominated atmosphere would only move the thermalization radius of the continuum to smaller radii (e.g., Roth et al. 2016), exacerbating the problem. A natural solution is for mass loading to reduce the final velocity of the outflow (Metzger \& Stone 2016), allowing it to be originally launched from smaller radii while matching the velocity seen in the absorption features.

The only other TDE in a quiescent galaxy that exhibits transient broad absorption features (in that case, $\mathrm{Mg}$ II $\lambda \lambda 2796$, 2803) with a comparable blueshift $\left(13,000 \mathrm{~km} \mathrm{~s}^{-1}\right)$ to AT2018zr (Chornock et al. 2014) is PS1-11af. The velocity of the absorbing material in PS1-11af is consistent with an outflow launched near the blackbody radius (Chornock et al. 2014). On the other hand, the line width of Mg II $\lambda \lambda 2796,2803$ $\left(\mathrm{FWHM} \approx 10,200 \mathrm{~km} \mathrm{~s}^{-1}\right)$ in PS1-11af is much broader than the absorption lines in AT2018zr (FWHM $\sim 3000 \mathrm{~km} \mathrm{~s}^{-1}$ for low-ionization lines and $\sim 10,000 \mathrm{~km} \mathrm{~s}^{-1}$ for high-ionization lines). The BALs are expected in the super-Eddington phase due to the wide range of gas velocities present in the outflow. This gives rise to a line width that is similar to the wind velocity (Strubbe \& Quataert 2011). The fact that the absorption lines in AT2018zr are narrower and detached from their emission peaks may be a viewing angle effect. For example, a few BALQSOs have been reported with detached absorption troughs (e.g., PG 1254+046; Hamann 1998). This may happen when our line of sight does not align with the wind such that the materials have already been substantially accelerated when it intersects our line of sight.

Although many TDE spectroscopic features resemble those of BALQSOs, at least a fraction of the BALQSOs have low Eddington ratios $\left(L / L_{\text {Edd }}<0.1\right.$; Grupe \& Nousek 2015), which must have a different mechanism to drive strong outflows. Indeed, the BALQSO wind is thought to be driven by line opacity in a partially ionized gas. In the line-driven wind framework proposed by Murray et al. (1995), dense gas that shields the soft X-ray near the hot QSO ionizing source is required to avoid overionization and drive the BAL outflows. Interestingly, the analysis of X-ray observations of AT2018zr also suggests some X-ray obscuring material residing outside the TDE photosphere (van Velzen et al. 2019). It is therefore also possible that the LoBAL in AT2018zr is powered by line opacity, though most BALQSOs are associated with black holes that are too massive $\left(M_{\mathrm{BH}}>10^{8} M_{\odot}\right)$ to tidally disrupt solar-type stars (Strubbe \& Quataert 2011).

Despite the TDE sample with UV spectroscopic observations still being small, the fraction of TDEs that exhibit BAL features in the UV (three out of four; Section 5.1) seems to be higher than the fraction $(\sim 20 \%)$ of BALQSOs, which is often attributed to the orientation effect in disk winds. This may suggest that the TDE outflows have different geometries than the QSO outflows and are less sensitive to viewing angle. Interestingly, the UV spectra of AT2018zr continue to support

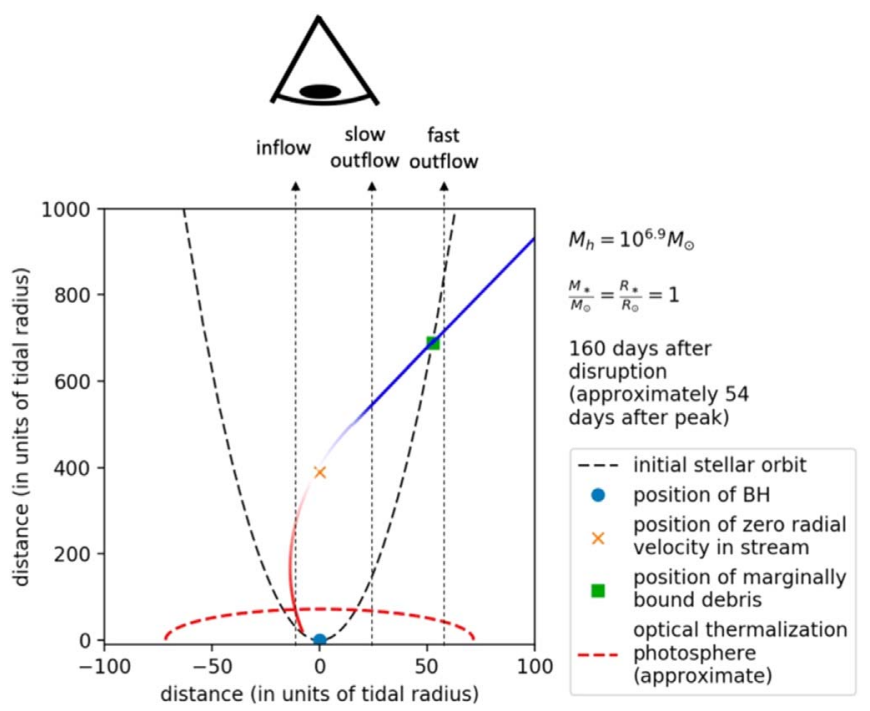

Figure 12. To-scale sketch of the stellar debris at the time corresponding to the spectral outflow signature. The red and blue curves trace out the outflowing and inflowing stellar debris streams, respectively. The viewing geometry is chosen to allow the unbound debris to provide the high-velocity absorption lines. However, in such a geometry, a wide range of gas velocities in the debris stream would intercept the optical photosphere, so we ultimately disfavor the debris stream as the origin of the high-velocity absorption lines. In order to identify the features of this diagram more clearly, we have stretched the horizontal distance axis by a factor of 5. We have referred to Coughlin et al. (2016) to determine the radial location of the portion of the stream at zero radial velocity.

the coincidence of BALs in X-ray-weak TDEs, as suggested by Yang et al. (2017). Future HST observations of TDEs are desirable for verifying the large fraction of BALs in TDEs and their potential anticorrelation with X-ray emission.

\subsubsection{Unbound Debris}

Although the blueshifted absorption features are known to form in disk winds, we also consider the unbound stellar debris as a possible absorber. In a tidal disruption, about half of the disrupted star gains enough energy to escape the black hole on a hyperbolic trajectory, reaching a terminal velocity of $11,000 \mathrm{~km} \mathrm{~s}^{-1}$ (Krolik et al. 2016). Simulations by Strubbe \& Quataert (2009) showed that the unbound debris irradiated by the accretion disk will produce emission lines at the optical-IR wavelengths. It is therefore possible that the rapid evolution in density $(\rho)$ and column density $(N)$ of the unbound debris, where $\rho \propto t^{-3}$ and $N \propto t^{-2}$, is causing the variability of the Balmer absorption seen on a timescale of a few days.

However, the orientation required for an observer to see the high-velocity unbound debris stream, as depicted in Figure 12, will also cover the slower portions of the stream, as well as the infalling bound stellar debris. The emergent spectra should, therefore, have broader line widths and even inflow signatures. However, these are clearly inconsistent with our observations, where the photosphere is eclipsed by material at a radial velocity of $15,500 \mathrm{~km} \mathrm{~s}^{-1}$ with a small dispersion of only $\sim 3000 \mathrm{~km} \mathrm{~s}^{-1}$.

\subsubsection{Physical Conditions}

Throughout the monitoring period, no significant acceleration or deacceleration in the absorbing material is detected, 
given the fact that the absorption features did not show any significant velocity offset between different epochs. Assuming the absorbing material has been traveling at constant velocity $\left(v=15,500 \mathrm{~km} \mathrm{~s}^{-1}\right)$ since peak light $\left(t_{\text {peak }}\right)$, it would have reached a distance of $\sim 5 \times 10^{15} \mathrm{~cm}$ by the time we first observed the $\mathrm{H} \beta$ absorption at $\Delta t \sim 30$ days. This distance is just outside of the continuum photosphere estimated from the blackbody fit (van Velzen et al. 2019).

Observations have revealed a handful of QSOs with hydrogen Balmer and/or metastable helium lines in their BAL systems. Comparisons of these observations with photoionization models have constrained the ionization parameters and densities of the outflows with the photoionization code CLOUDY, which, in turn, can constrain the kinetic luminosities of the outflows. Below, we summarize the physical conditions that give rise to the hydrogen Balmer and helium lines based on these works. In particular, we reference the values derived with CLOUDY version 17.00 (Ferland et al. 2013) by Hamann et al. (2019), given the presence of similar species in the transient absorber in PG 1411+442. In their calculation, Hamann et al. (2019) employed plane-parallel clouds with solar abundances and an input continuum with a broken power-law form $f_{\nu} \propto \nu^{\alpha}$, where $\alpha_{U V}=-0.5$ and -0.9 . In general, the TDE disk spectrum is thought to be harder than that of an AGN, given the smaller size of the accretion disk. Although this high-energy tail of X-ray emission is rarely seen in optically selected TDEs, it has been suggested that the HeII4686 and Bowen emission lines may be indirect evidence for extreme UV or reprocessed X-ray emission (e.g., Blagorodnova et al. 2019; Leloudas et al. 2019).

Strong helium transitions from the metastable $2 \mathrm{~s}$ level occur at $\lambda 3889$ and $\lambda 10830$, which correspond to transitions from the metastable state to the $3 p$ and $2 p$ states, respectively. Although once considered a rare phenomenon, Liu et al. (2015) found a high association rate between $\mathrm{He} \mathrm{I}^{*} \lambda 3889$ and the Mg II $\lambda \lambda 2796,2803$ doublet among BALQSOs with high-S/ $\mathrm{N}$ spectra $(93 \%$ at $\mathrm{S} / \mathrm{N} \gtrsim 35)$. Leighly et al. (2011) demonstrated that the $\mathrm{He} \mathrm{I}^{*} \lambda 3889$ and $\mathrm{He} \mathrm{I}^{*} \lambda 10830$ lines can serve as a powerful diagnostic of high column density outflow. This is due to the large $\lambda f_{i k}$ ratio $^{16}$ of 23.3 , which makes the He I* lines sensitive to a wide range of high column densities $\left(\tau \propto N_{\text {ion }} \lambda f_{i k}\right)$ before saturating. Unfortunately, we are unable to carry out a similar analysis due to the $\mathrm{He}{ }^{*} \lambda 10830$ line falling outside our spectroscopic coverage. The presence of He I ${ }^{*} \lambda 5876$ provides additional constraints on the ionized gas. Unlike $\mathrm{He} \mathrm{I}^{*} \lambda 3889$, He I ${ }^{*} \lambda 5876$ arises from an energy state that is not metastable and is readily depopulated by permitted radiative decays via the $\lambda 10830$ transition when the gas density is low. Photoionization calculations have shown that a gas density of $\log \left(n_{\mathrm{H}} / \mathrm{cm}^{-3}\right) \gtrsim 7$ and an ionized column density of $\log \left(N_{\mathrm{H}} / \mathrm{cm}^{-2}\right) \gtrsim 23$ are required to produce $\tau_{0}(5876) \sim 0.1$ (Hamann et al. 2019). The inferred high density and column density are required to populate the highly excited states and form the He I ${ }^{*} \lambda 5876$ absorption line regardless of the exact ionizing spectrum.

The blueshift velocity of AT2018zr is the highest among QSOs with Balmer BALs (Zhang et al. 2015). Behind the hydrogen recombination front, the partially ionized regions that give rise to the $\mathrm{H}$ Balmer absorption lines usually have high densities (typically $n_{e} \sim 10^{6-9} \mathrm{~cm}^{-3}$; Zhang et al. 2015, and

\footnotetext{
${ }^{16}$ Here $\lambda f_{i k}$ (wavelength of the transition $\times$ oscillator strength) is a measurement of the optical depth of an absorption line (see Equation (3)).
}

references therein). Our analysis of the $\mathrm{H}$ Balmer absorption lines has shown that even the high-order hydrogen Balmer transitions are saturated. Photoionization modeling with CLOUDY suggests that a density of $\log \left(n_{H} / \mathrm{cm}^{-3}\right) \gtrsim 6.5$ and a column density of $\log \left(N_{H} / \mathrm{cm}^{-2}\right) \gtrsim 23.2$ are required to produce $\tau_{0}(\mathrm{H} \gamma) \gtrsim 1.3$ regardless of the ionization parameter $U$ (Hamann et al. 2019).

With the above estimation of density and column density, a cloud size of $10^{16} \mathrm{~cm}$ can be inferred from $N_{\mathrm{H}} / n_{\mathrm{H}}$ (assuming $\log \left(n_{\mathrm{H}} / \mathrm{cm}^{-3}\right)=7$ and $\left.\log \left(N_{H} / \mathrm{cm}^{-2}\right) \gtrsim 23\right)$. Assuming a spherical cloud, we estimate a total mass of $4.4 \times 10^{-3} M_{\odot}$ and a kinetic energy of $10^{49} \mathrm{erg}$. The mass and kinetic energy of the outflow in AT2018zr are about an order of magnitude greater than that in ASASSN-14li, which is known to host an outflow with comparable velocity $\left(12,000-36,000 \mathrm{~km} \mathrm{~s}^{-1}\right)$, as derived from radio observations (Alexander et al. 2016). Note that radio emission, which is expected as the outflow shocks against the circumnuclear matter (CNM), is not detected in AT2018zr at a $3 \sigma$ upper limit of $10^{37} \mathrm{erg} \mathrm{s}^{-1}$ at $10 \mathrm{GHz}$ (van Velzen et al. 2019). While the HST data show unambiguous evidence for the presence of an outflow, the nondetection at the radio frequency may be due to the radio signal being buried by the dense CNM.

Detailed, accurate characterization of the broad emission and absorption lines in the UV is necessary for robust estimation of the transient absorber properties (e.g., column density). In particular, the column density and density are critical parameters to measure the kinetic luminosity, which can be used to examine different TDE outflow models and assess their importance in the context feedback in galaxy evolution.

\section{Conclusion}

We report the results of our analysis of multi-epoch UV and optical spectra of the TDE AT2018zr observed between 2018 March and December. The wide wavelength coverage of the HST STIS UV spectra $(\sim 1150-3000 \AA)$, together with the Keck LRIS spectra $(\sim 3500-9000 \AA)$, allows us to identify highly blueshifted $\left(15,500 \mathrm{~km} \mathrm{~s}^{-1}\right.$, or $\left.0.05 c\right)$ BALs in the UV and optical, including the high- and low-ionization transitions seen in LoBAL QSOs.

In this BAL system, we identified the first hydrogen Balmer and metastable He I transitions, which are known to be rare in QSOs, in a TDE. We conclude that this BAL system is more likely the result of a high-velocity outflow launched by TDE accretion flows, instead of the unbound debris. Given that the presence of broad UV absorption lines is more common in TDEs (three out of four) than in QSOs, this may suggest that the outflow launching mechanisms in TDEs are less subjective to orientation effects than those in QSOs.

Our curve-of-growth analysis shows that even the high-order hydrogen Balmer absorption lines are saturated, and the absorbing material only covers the background source partially. By comparing with photoionization models for AGNs, we conclude that the ionized gas must be characterized by a high density $\left(\log \left(n_{\mathrm{H}} / \mathrm{cm}^{-3}\right) \gtrsim 7\right)$ and high column (log $\left.\left(N_{\mathrm{H}} / \mathrm{cm}^{-2}\right) \gtrsim 23\right)$ based on the detection of He I ${ }^{*} \lambda 3889$ and $\mathrm{He} \mathrm{I}^{*} \lambda 5876$. The inference of high density and high column is not very sensitive to the exact ionizing spectrum. In order to give rise to the $\mathrm{H}$ Balmer absorption, the partially ionized gas behind the hydrogen recombination front must also have high column densities. More UV and optical spectroscopic observations of TDEs will allow detailed photoionization modeling to 
assess whether TDE outflows provide significant "feedback" in the context of galaxy evolution.

Using a radiative transfer model, we show that the emission profile seen in expanding TDE outflows (Roth \& Kasen 2018) is similar to the flat-topped $\mathrm{H} \alpha$ line shape in our observations. We find that the spherical outflow model alone, with a maximum velocity of $1.75 \times 10^{4} \mathrm{~km} \mathrm{~s}^{-1}$ close to the velocity of the aforementioned BAL, provides a satisfactory fit to the observed line profile. While the elliptical disk model has been invoked to interpret emission lines in several TDEs, it requires stringent conditions in order to produce the line shape in AT2018zr. Furthermore, the implied disk size from the elliptical disk model is 2 orders of magnitude larger than the stream self-intersection radius, which is difficult to explain from the perspective of debris stream dynamics.

We report the appearance of narrow $\mathrm{He}$ II $\lambda 4686, \mathrm{H} \alpha$, and $\mathrm{H} \beta$ emission lines in the late-time optical spectra of AT2018zr. The line widths of these transitions imply a virial radius on the order of $10^{17} \mathrm{~cm}$. We suggest that the presence of He II $\lambda 4686$ and $\mathrm{N}$ III $\lambda 4640$ emission in later observations may be driven by the temperature increase in the late-time UV and optical continuum.

The TDE spectra are thought to be rather featureless; hence, the spectroscopic analysis has been focused on the emission lines in the past. We emphasize that subtle features, such as the highly blueshifted H Balmer absorption lines in AT2018zr, may also be present in TDE spectra as a result of outflows. High-S/N spectra with broad wavelength coverage are critical for identifying these absorption features in the UV and optical. We also recommend monitoring future TDEs with spectroscopy on a weekly basis, since, as we have shown with AT2018zr, the spectroscopic features-both emission and absorption-are variable on such a timescale.

T.H. thanks Fred Hamann for sharing a copy of his draft prior to publication. T.H. is grateful to Jane Dai and Enrico Ramirez-Ruiz for helpful discussions. The authors would like to thank the anonymous referee for suggestions that greatly improved the clarity of the paper. The UCSC team is supported in part by NSF grant AST-1518052, the Gordon \& Betty Moore Foundation, the Heising-Simons Foundation, and a fellowship from the David and Lucile Packard Foundation to R.J.F. S.V. acknowledges support from a Raymond and Beverley Sackler Distinguished Visitor Fellowship and thanks the host institute, the Institute of Astronomy, where this work was concluded. S.G is supported in part by NSF grant 1454816. S.V. also acknowledges support by the Science and Technology Facilities Council (STFC) and the Kavli Institute for Cosmology, Cambridge. N.B. acknowledges that this work is part of the research program VENI, with project No. 016.192.277, which is (partly) financed by the Netherlands Organisation for Scientific Research (NWO). M.R.S. is supported by the National Science Foundation Graduate Research Fellowship Program under grant No. 1842400. A. G.-Y. is supported by the EU via ERC grant No. 725161, the ISF, the BSF Transformative program, and a Kimmel award. The research of Y.Y. is supported through a Benoziyo Prize Postdoctoral Fellowship. Y.Y. thanks support astronomers at La Palma for assisting the WHT observation in service mode.

Based on observations made with the NASA/ESA Hubble Space Telescope, obtained from the Data Archive at the Space Telescope Science Institute, which is operated by the
Association of Universities for Research in Astronomy, Inc., under NASA contract NAS 5-26555. The results made use of the Discovery Channel Telescope at Lowell Observatory. Lowell is a private, nonprofit institution dedicated to astrophysical research and public appreciation of astronomy and operates the DCT in partnership with Boston University, the University of Maryland, the University of Toledo, Northern Arizona University, and Yale University. Some of the data presented herein were obtained at the W. M. Keck Observatory, which is operated as a scientific partnership among the California Institute of Technology, the University of California, and the National Aeronautics and Space Administration. The Observatory was made possible by the generous financial support of the W. M. Keck Foundation. The authors wish to recognize and acknowledge the very significant cultural role and reverence that the summit of Maunakea has always had within the indigenous Hawaiian community. We are most fortunate to have the opportunity to conduct observations from this mountain. The William Herschel Telescope is operated on the island of La Palma by the Isaac Newton Group of Telescopes in the Spanish Observatorio del Roque de los Muchachos of the Instituto de Astrofísica de Canarias. The ACAM spectroscopy was obtained as part of OPT/2018A/ 017. The results in this work are based on observations obtained with the Samuel Oschin Telescope 48 inch and the 60 inch telescope at the Palomar Observatory as part of the Zwicky Transient Facility (ZTF) project. The ZTF is supported by the National Science Foundation under grant No. AST1440341 and a collaboration including Caltech, IPAC, the Weizmann Institute for Science, the Oskar Klein Center at Stockholm University, the University of Maryland, the University of Washington, Deutsches Elektronen-Synchrotron and Humboldt University, Los Alamos National Laboratories, the TANGO Consortium of Taiwan, the University of Wisconsin at Milwaukee, and Lawrence Berkeley National Laboratories. Operations are conducted by COO, IPAC, and UW. The SEDm is based upon work supported by the National Science Foundation under grant No. 1106171.

\section{ORCID iDs}

T. Hung (1) https://orcid.org/0000-0002-9878-7889

S. B. Cenko (1) https://orcid.org/0000-0003-1673-970X

Nathaniel Roth (1) https://orcid.org/0000-0002-6485-2259

S. Gezari (i) https://orcid.org/0000-0003-3703-5154

S. Veilleux (i) https://orcid.org/0000-0002-3158-6820

Sjoert van Velzen (1) https://orcid.org/0000-0002-3859-8074

C. Martin Gaskell (i) https://orcid.org/0000-0003-4888-2009

N. Blagorodnova (1) https://orcid.org/0000-0003-0901-1606

Lin Yan (ㄴ) https://orcid.org/0000-0003-1710-9339

M. J. Graham (1) https://orcid.org/0000-0002-3168-0139

J. S. Brown (1) https://orcid.org/0000-0002-1885-6419

Sara Frederick (1) https://orcid.org/0000-0001-9676-730X

Avishay Gal-Yam (1) https://orcid.org/0000-0002-3653-5598

S. Schulze iㅏ https://orcid.org/0000-0001-6797-1889

G. Dimitriadis (10) https://orcid.org/0000-0001-9494-179X

Thomas Kupfer (1) https://orcid.org/0000-0002-6540-1484

David L. Shupe (1) https://orcid.org/0000-0003-4401-0430

Frank J. Masci (다 https://orcid.org/0000-0002-8532-9395

Reed Riddle (1) https://orcid.org/0000-0002-0387-370X

Maayane T. Soumagnac (i) https://orcid.org/0000-00016753-1488 


\section{References}

Alexander, K. D., Berger, E., Guillochon, J., Zauderer, B. A., \& Williams, P. K. G. 2016, ApJL, 819, L25

Aoki, K. 2010, PASJ, 62, 1333

Arav, N., Brotherton, M. S., Becker, R. H., et al. 2001, ApJ, 546, 140

Bellm, E. C., Kulkarni, S. R., Graham, M. J., et al. 2019, PASP, 131, 018002 Bennett, C. L., Larson, D., Weiland, J. L., et al. 2013, ApJ, 208, 20

Blagorodnova, N., Cenko, S. B., Kulkarni, S. R., et al. 2019, ApJ, 873, 92

Blagorodnova, N., Neill, J. D., Walters, R., et al. 2018, PASP, 130, 035003

Brotherton, M. S., Tran, H. D., Becker, R. H., et al. 2001, ApJ, 546, 775

Brown, J. S., Kochanek, C. S., Holoien, T. W.-S., et al. 2018, MNRAS, 473, 1130

Cao, R., Liu, F. K., Zhou, Z. Q., Komossa, S., \& Ho, L. C. 2018, MNRAS, 480, 2929

Cardelli, J. A., Clayton, G. C., \& Mathis, J. S. 1989, ApJ, 345, 245

Cenko, S. B., Cucchiara, A., Roth, N., et al. 2016, ApJL, 818, L32

Chen, K., Halpern, J. P., \& Filippenko, A. V. 1989, ApJ, 339, 742

Chornock, R., Berger, E., Gezari, S., et al. 2014, ApJ, 780, 44

Coughlin, E. R., Nixon, C., Begelman, M. C., \& Armitage, P. J. 2016, MNRAS, 459, 3089

Curd, B., \& Narayan, R. 2019, MNRAS, 483, 565

Dai, L., McKinney, J. C., \& Miller, M. C. 2015, ApJL, 812, L39

Dai, L., McKinney, J. C., Roth, N., Ramirez-Ruiz, E., \& Miller, M. C. 2018, ApJL, 859, L20

Eracleous, M., \& Halpern, J. P. 1994, ApJS, 90, 1

Eracleous, M., \& Halpern, J. P. 2003, ApJ, 599, 886

Eracleous, M., Livio, M., Halpern, J. P., \& Storchi-Bergmann, T. 1995, ApJ, 438,610

Evans, C. R., \& Kochanek, C. S. 1989, ApJL, 346, L13

Ferland, G. J., Porter, R. L., van Hoof, P. A. M., et al. 2013, RMxAA, 49, 137

Gallegos-Garcia, M., Law-Smith, J., \& Ramirez-Ruiz, E. 2018, ApJ, 857, 109

Ganguly, R., Brotherton, M. S., Cales, S., et al. 2007, ApJ, 665, 990

Gaskell, C. M. 1982, ApJ, 263, 79

Gaskell, C. M. 2009, NewAR, 53, 140

Gaskell, C. M. 2017, MNRAS, 467, 226

Gaskell, C. M., \& Rojas Lobos, P. A. 2017, MNRAS, 438, L36

Gehrels, N., Chincarini, G., Giommi, P., et al. 2004, ApJ, 611, 1005

Gezari, S., Chornock, R., Rest, A., et al. 2012, Natur, 485, 217

Graham, M. J., Kulkarni, S. R., Bellm, E. C., et al. 2019, PASP, 131, 078001

Grupe, D., \& Nousek, J. A. 2015, AJ, 149, 85

Hamann, F. 1998, ApJ, 500, 798

Hamann, F., Tripp, T. M., Rupke, D. S. N., \& Veilleux, S. 2019, MNRAS, 487, 5041

Hewett, P. C., \& Foltz, C. B. 2003, AJ, 125, 1784

Holoien, T. W.-S., Huber, M. E., Shappee, B. J., et al. 2018, arXiv:1808.02890

Holoien, T. W.-S., Kochanek, C. S., Prieto, J. L., et al. 2016a, MNRAS, 463, 3813

Holoien, T. W.-S., Kochanek, C. S., Prieto, J. L., et al. 2016b, MNRAS, 455, 2918

Holoien, T. W.-S., Prieto, J. L., Bersier, D., et al. 2014, MNRAS, 445, 3263

Hung, T., Gezari, S., Blagorodnova, N., et al. 2017, ApJ, 842, 29

Hung, T., Gezari, S., Cenko, S. B., et al. 2018, ApJS, 238, 15

Jeffery, D. J., \& Branch, D. 1990, in Jerusalem Winter School for Theoretical Physics, ed. J. C. Wheeler, T. Piran, \& S. Weinberg (Singapore: World Scientific), 149
Ji, T., Wang, T.-G., Zhou, H.-Y., \& Wang, H.-Y. 2012, RAA, 12, 369 Jiang, Y.-F., Stone, J., \& Davis, S. W. 2017, arXiv:1709.02845

Kara, E., Dai, L., Reynolds, C. S., \& Kallman, T. 2018, MNRAS, 474, 3593

King, A. R., \& Pounds, K. A. 2003, MNRAS, 345, 657

Kochanek, C. S. 2016, MNRAS, 458, 127

Krolik, J., Piran, T., Svirski, G., \& Cheng, R. M. 2016, ApJ, 827, 127

Landt, H., Ward, M. J., Elvis, M., \& Karovska, M. 2014, MNRAS, 439, 1051

Leighly, K. M., Dietrich, M., \& Barber, S. 2011, ApJ, 728, 94

Leloudas, G., Dai, L., Arcavi, I., et al. 2019, arXiv:1903.03120

Liu, F. K., Zhou, Z. Q., Cao, R., Ho, L. C., \& Komossa, S. 2017, MNRAS, 472, L99

Liu, W.-J., Zhou, H., Ji, T., et al. 2015, ApJS, 217, 11

Lodato, G., \& Rossi, E. M. 2011, MNRAS, 410, 359

Mathews, W. G. 1982, ApJ, 258, 425

Metzger, B. D., \& Stone, N. C. 2016, MNRAS, 461, 948

Miller, J. M., Kaastra, J. S., Miller, M. C., et al. 2015, Natur, 526, 542

Miller, M. C. 2015, ApJ, 805, 83

Murray, N., Chiang, J., Grossman, S. A., \& Voit, G. M. 1995, ApJ, 451, 498

Ohsuga, K., Mori, M., Nakamoto, T., \& Mineshige, S. 2005, ApJ, 628, 368

Oke, J. B., Cohen, J. G., Carr, M., et al. 1995, PASP, 107, 375

Pasham, D. R., \& van Velzen, S. 2018, ApJ, 856, 1

Phinney, E. S. 1989, in IAU Symp. 136, The Center of the Galaxy, ed. M. Morris (Berlin: Springer), 543

Rees, M. J. 1988, Natur, 333, 523

Reichard, T. A., Richards, G. T., Schneider, D. P., et al. 2003, AJ, 125, 1711

Richards, G. T., Vanden Berk, D. E., Reichard, T. A., et al. 2002, AJ, 124, 1

Roth, N., \& Kasen, D. 2018, ApJ, 855, 54

Roth, N., Kasen, D., Guillochon, J., \& Ramirez-Ruiz, E. 2016, ApJ, 827, 3

Rudy, R. J., Foltz, C. B., \& Stocke, J. T. 1985, ApJ, 288, 531

Sąowski, A., Narayan, R., McKinney, J. C., \& Tchekhovskoy, A. 2014, MNRAS, 439, 503

Schlafly, E. F., \& Finkbeiner, D. P. 2011, ApJ, 737, 103

Schulze, A., Misawa, T., Zuo, W., \& Wu, X.-B. 2018, ApJ, 853, 167

Shi, X., Zhou, H., Shu, X., et al. 2016, ApJ, 819, 99

Strateva, I. V., Strauss, M. A., Hao, L., et al. 2003, AJ, 126, 1720

Strubbe, L. E., \& Quataert, E. 2009, MNRAS, 400, 2070

Strubbe, L. E., \& Quataert, E. 2011, MNRAS, 415, 168

Sun, L., Zhou, H., Ji, T., et al. 2017, ApJ, 838, 88

Svirski, G., Piran, T., \& Krolik, J. 2017, MNRAS, 467, 1426

Trump, J. R., Hall, P. B., Reichard, T. A., et al. 2006, ApJS, 165, 1

Tucker, M. A., Huber, M., Shappee, B. J., et al. 2018, ATel, 11473

Ulmer, A. 1999, ApJ, 514, 180

van Velzen, S., Anderson, G. E., Stone, N. C., et al. 2016, Sci, 351, 62

van Velzen, S., Gezari, S., Cenko, S. B., et al. 2019, ApJ, 872, 198

Vanden Berk, D. E., Richards, G. T., Bauer, A., et al. 2001, AJ, 122, 549

Veilleux, S., Meléndez, M., Tripp, T. M., Hamann, F., \& Rupke, D. S. N. 2016, ApJ, 825, 42

Wevers, T., van Velzen, S., Jonker, P. G., et al. 2017, MNRAS, 471, 1694

Wiese, W. L., \& Fuhr, J. R. 2009, JPCRD, 38, 565

Wu, S., Coughlin, E. R., \& Nixon, C. 2018, MNRAS, 478, 3016

Yang, C., Wang, T., Ferland, G. J., et al. 2017, ApJ, 846, 150

Zhang, S., Zhou, H., Shi, X., et al. 2015, ApJ, 815, 113 